\title{
GOAL-ORIENTED ERROR ESTIMATION AND MESH ADAPTATION FOR TRACER TRANSPORT MODELLING
}

\author{
THIS ARTICLE IS A NON-PEER REVIEWED PREPRINT SUBMITTED TO EARTHARXIV. IT HAS ALSO BEEN \\ SUBMITTED TO THE JOURNAL COMPUTER AIDED DESIGN.
}

\author{
Joseph G. Wallwork \\ Department of Earth Science and Engineering \\ Imperial College London, UK \\ j.wallwork16@imperial.ac.uk
}

\author{
David A. Ham \\ Department of Mathematics \\ Imperial College London, UK
}

\author{
Nicolas Barral \\ University of Bordeaux, CNRS, Bordeaux INP \\ IMB, UMR 5251, 33400, Talence, France \\ INRIA, IMB, UMR 5251, 33400, Talence, France \\ Matthew D. Piggott \\ Department of Earth Science and Engineering \\ Imperial College London, UK
}

January 26, 2021

\begin{abstract}
This paper applies metric-based mesh adaptation methods to advection-dominated tracer transport modelling problems in two and three dimensions, using the finite element package Firedrake. In particular, the mesh adaptation methods considered are built upon goal-oriented estimates for the error incurred in evaluating a diagnostic quantity of interest $(Q o I)$. In the motivating example of modelling to support desalination plant outfall design, such a QoI could be the salinity at the plant inlet, which could be negatively impacted by the transport of brine from the plant's outfall. Four approaches are considered, one of which yields isotropic meshes. The focus on advection-dominated problems means that flows are often anisotropic; thus, three anisotropic approaches are also considered. Meshes resulting from each of the four approaches yield solutions to the tracer transport problem which give better approximations to QoI values than uniform meshing, for a given mesh size. The methodology is validated using an existing 2D tracer transport test case with a known analytical solution. Goaloriented meshes for an idealised time-dependent desalination outfall scenario are also presented.
\end{abstract}

Keywords Anisotropy $\cdot$ Mesh adaptation $\cdot$ Error estimation $\cdot$ Desalination outfall $\cdot$ Firedrake

\section{Introduction}

Coastal ocean modelling is primarily concerned with understanding the hydrodynamics of coastal processes through numerical simulations. This includes the transport of passive and active tracers such as sediment, pollutants and salinity. Such tracer fields are typically modelled using equations of advection-diffusion type. In some cases, such as sediment transport, there is a two-way coupling with the hydrodynamics - the active case. In other cases, such as with pollutants suspended in water, it is sufficient to model the tracer as passive, with its transport being purely driven by the flow velocity. For problems in either category, there is often a relevant quantity of interest (QoI) which a user of a model wishes to deduce from the solution field. For sediment transport problems, this may be the erosion of a particular bank. For pollutants, it might be the concentration in a region of particular environmental importance. The scenario considered in this paper is that of a desalination plant, where the salinity at the inlet pipe is a useful QoI when using modelling to support the design process of locating the inlet and outfall locations. The goal of this work is to generate adaptive meshes which minimise the error incurred in evaluating the chosen QoI. This in turn optimises the speed vs accuracy of the calculation required at each step of an iterative design optimisation process. 
Mesh adaptation typically requires two ingredients: an error estimator (defined on the current mesh) and a method which uses this error estimator to obtain an adapted mesh. Each of these components involves a design choice.

In line with the above discussion, the error estimation approach used in this work is framed around the QoI error. The class of error estimates associated with the QoI error are termed goal-oriented (or goal/output-based) in the literature. Many goal-oriented error estimates are based on the a posteriori error result established in [1, 2] - the dual-weighted residual (DWR). This result gives rise to first and second order error estimates for the QoI error, in terms of residuals and errors related to forward and adjoint equations.

By considering local contributions to the DWR error estimator, it is possible to identify regions of the domain where the use of increased mesh resolution will reduce the overall QoI error. Similarly, regions of the domain may be determined where it is admissible to decrease mesh resolution, with little impact on accuracy. Thus, goal-oriented mesh adaptation routines are often built upon element-wise error indicator fields. Dual weighted residual error estimation has been used to drive effective meshing strategies for a number of tracer transport applications (such as [3, 4, 5, 6, 7]). This work includes a comparison of three such approaches, as well as one based on an alternative error estimate. It also includes an extension of the goal-oriented framework to the time-dependent case.

In terms of the mesh adaptation model, element-wise error indicators lend themselves well to hierarchical $h$-adaptation methods which refine on an element-by-element basis, such as quad-tree and oct-tree type methods (see [2], for example). In these methods, mesh elements are refined or coarsened based on whether the local error indicator value meets pre-specified thresholds. Hierarchical mesh adaptation methods have been combined with an adaptive polynomial degree ( $p$-adaptation), yielding hybrid $h p$-adaptation methods. See [6] for an example of goal-oriented $h p$-adaptation in the context of tracer transport modelling.

In general, however, hierarchical adaptation methods do not incorporate directionality, producing meshes whose elements are as isotropic as those in the original mesh. We focus on advection-dominated tracer transport, wherein the fluid flows (and/or regions of high tracer concentration variation) are anisotropic. Using appropriate anisotropic meshes, these direction-dependent features may be accurately resolved using relatively few (spatial) degrees of freedom (DoFs). As such, it makes sense to use a mesh adaptation method which can also account for this anisotropy. Where 'isotropic' adaptation strategies allow only to modify the size of an element, anisotropic strategies also account for its shape and orientation. The anisotropic meshing approach used in this work is based upon a metric-based framework [8, 9, 10, 11, 12]. Riemannian metric fields act as continuous analogues for (inherently discrete) meshes [11].

Assuming that mesh adaptation is to be driven by metric fields, one of the main research questions of this paper is how to effectively construct such a metric from goal-oriented error estimates. In particular, effective approaches to anisotropic metric construction are sought.

The literature contains a number of notable efforts in the development of goal-oriented metrics. The simplest approach is to multiply the identity matrix by an appropriately scaled projection of the (scalar) DWR error (for examples, see [5, 13]). Whilst straightforward to construct, the resulting metrics do not account for anisotropy.

The first documented use of dual-weighted residual error estimation techniques for constructing anisotropic metrics is presented in [3]. There, an error result is derived which bounds the interpolation error of a scalar field on a mesh element by an expression involving element anisotropy coefficients and the Hessian of the field. The eigenvalues of a Hessian matrix are modified in order to enforce element sizes and include anisotropic stretching factors, as dictated by local error indicators. Hessian matrices are useful because they contain information on anisotropy. The approach was extended to higher order elements and flows with strongly anisotropic features in [5]. An element-based formulation is used, which fits naturally with the fact that the DWR yields element-based error indicators.

A simple approach which does not use the DWR estimator directly is that introduced in [14]. By reinterpreting the error in the adjoint solution as an interpolation error, the authors of [14] argue for a formulation in which the Hessian of the approximate adjoint solution may be weighted by the strong residual of the forward equation.

Like the approach of [14], the authors of [15] again reinterpret errors, leading to another Hessian-based formulation which involves the combination of anisotropic metrics for both domain interior and domain boundary. Unlike those described above, this approach is not based on the (a posteriori) DWR error result; instead it is based upon an a priori error result, first derived in [15]. The formulation was extended to the time-dependent case in [16, 17].

This paper builds upon the work of [13] and [18], which implemented goal-oriented mesh adaptation techniques in the Python-based finite element package, Firedrake [19]. The four goal-oriented mesh adaptation routines implemented in those works are compared in the context of an analytical test case. This is the first time these four approaches have been compared in the same software package. Simplifications in the original implementations are overcome and stabilisation terms are properly accounted for. As mentioned above, a novelty of this paper is its extension of the goal-oriented 
framework to the time-dependent case. This extension is a necessary step towards solving realistic tracer transport problems.

The implementation makes use of the tracer transport modelling framework developed in the Thetis ocean modelling project [20]. Software versions used for numerical experiments presented in this work are archived through Zenodo as [21, 22]. The simulation code used to generate all results is archived as [23].

The remainder of the paper is arranged as follows. The metric-based mesh adaptation strategy is introduced in Section 2 Section 3 applies goal-oriented error estimation to an advection-diffusion problem. In addition, four strategies for the construction of goal-oriented metrics are presented and later used for numerical experiments in Sections 4 and 5. Section 4 considers the steady-state case, including validation experiments based on the TELEMAC-2D 'Point Discharge with Diffusion' test case, for which an analytical solution exists [24]. The 3D case is also considered. Section 5 considers the extension to time-dependent problems, presenting meshes arising in an idealised desalination plant outfall scenario. Finally, conclusions are drawn in Section 6, with potential future work discussed.

\section{Metric-Based Mesh Adaptation}

As discussed in Section 1 this work utilises a metric-based approach. This means that mesh adaptation processes are driven by Riemannian metric fields of dimension $n \times n$, where $\Omega \subset \mathbb{R}^{n}$ is the PDE domain. A Riemannian metric field, or metric, denoted $\mathcal{M}=\{\underline{M}(\mathbf{x})\}_{\mathbf{x} \in \Omega}$, is a collection of symmetric positive-definite (SPD) linear forms defined pointwise. Encoded within the metric is local information on distances, from which anisotropic edge lengths and element volumes can be derived.

Metric-based mesh adaptation was first introduced in [8]. It uses a Riemannian metric space within the mesher in order to compute the necessary geometrical quantities. The aim of the adaptation process is to generate a unit mesh with respect to this Riemannian metric space. That is, all mesh elements are in some sense close to being isotropic, when viewed in the metric space. A major advantage of the metric-based approach is that it enables control of mesh anisotropy, meaning that not only element size, but also shape and orientation may be dictated.

Given an error estimator, we seek a mesh of the spatial domain such that the numerical solution of a PDE either: (a) achieves a certain level of error [9, 14]; or (b) minimises the interpolation error for a given number of mesh vertices [15, 25]. In this work we follow the latter approach. For this, we need to establish the error estimator and express it as a metric.

Throughout this paper, we use the notation $\mathcal{H}$ when referring to meshes and $K$ to denote elements thereof. Occasionally, a cell size function $h$ is mentioned as a subscript, $\mathcal{H}_{h}$, if instructive. The edge set of element $K$ is denoted $\partial K$, with outward normal vectors $\widehat{\mathbf{n}}_{K}$. The indicator function which is unity on element $K$ and zero elsewhere is denoted $\mathbb{1}_{K}$.

\subsection{Hessian-Based Metric}

Suppose $u$ is a (sufficiently smooth) scalar field of interest whose Hessian may be approximated as $\underline{\boldsymbol{H}}$. For any $p \in[1, \infty)$, the interpolation error associated with a piecewise linear and continuous $(\mathbb{P} 1)$ approximation $\Pi_{h} u$ is related to $\underline{\boldsymbol{H}}$ by the upper bound [10]

$$
\left\|u-\Pi_{h} u\right\|_{\mathcal{L}_{p}(\Omega)} \leq n N^{-\frac{2}{n}}\left(\int_{\Omega} \operatorname{det}(|\underline{\boldsymbol{H}}|)^{\frac{p}{2 p+n}} \mathrm{~d} x\right)^{\frac{2 p+n}{n p}},
$$

provided that we have a unit mesh with respect to a metric of complexity $N>0$. Metric complexity is the continuous analogue of the mesh vertex count, defined by [11]

$$
\mathcal{C}(\mathcal{M}):=\int_{\Omega} \sqrt{\operatorname{det}(\underline{\boldsymbol{M}}(\mathbf{x}))} \mathrm{d} x .
$$

Since $\underline{\boldsymbol{H}}$ is symmetric, it has an orthogonal eigen-decomposition, $\underline{\boldsymbol{H}}=\underline{\boldsymbol{V}} \underline{\boldsymbol{\Lambda}} \underline{\boldsymbol{V}}^{T}$, meaning it makes sense to take the absolute value as

$$
|\underline{\boldsymbol{H}}|=\underline{\boldsymbol{V}}|\underline{\boldsymbol{\Lambda}}| \underline{\boldsymbol{V}}^{T} \text {. }
$$

Taking the absolute value implies that $|\underline{H}|$ is SPD and is therefore a valid metric. The justification for doing so is that we are primarily interested in the magnitude of errors, as opposed to their sign.

In practice, $u$ is only known in a discrete sense, meaning its Hessian must be approximated using a recovery technique, typically involving the solution of an auxiliary PDE. In this work, double $\mathcal{L}_{2}$ projection is applied (see [9] for details).

For further details on Hessian-based mesh adaptation, see [8, 9, 10, 11, 26]. 


\section{$2.2 \mathcal{L}_{p}$-Normalisation}

For a metric to be applicable to any problem, it is necessary to scale it appropriately. The scaling process also allows a degree of control over various properties of the resulting meshes, such as the number of vertices and the extent to which the meshes have a multi-scale nature.

An $\mathcal{L}_{p}$-normalisation strategy of order $p \in[1, \infty)$ for a metric $\mathcal{M}$ is typically governed by either a target interpolation error level, $\epsilon>0$, or a target metric complexity, $N>0$ [10]. In the former case, a metric is sought which admits interpolation error smaller than $\epsilon$. Here, we use the latter approach, whereby we seek a metric whose complexity matches the target value, $N$.

For time-independent problems, this constraint is met by applying the formula [10]

$$
\mathcal{M}_{\mathcal{L}_{p}}:=N^{\frac{2}{n}}\left(\int_{\Omega} \operatorname{det}(\mathcal{M})^{\frac{p}{2 p+n}} \mathrm{~d} x\right)^{-\frac{2}{n}} \operatorname{det}(\mathcal{M})^{\frac{-1}{2 p+n}} \mathcal{M}
$$

By specifying larger values for the target complexity $N$ in (4), we allow heightened mesh size in return for reduced interpolation error.

Taking the limit $p \rightarrow \infty$ yields the strategy referred to as $\mathcal{L}_{\infty}$ normalisation. Whilst commonly used, the $\mathcal{L}_{\infty}$ normalisation strategy focuses resolution around only the most prominent features, using very high levels of mesh refinement surrounding them. Lower order $\mathcal{L}_{p}$ normalisation strategies yield more multi-scale meshes, with $p \approx 1$ able to capture all scales.

In the case of time-dependent problems, the normalisation approach should be applied over the time period, $(0, T]$, too. Assuming a timestep, $\Delta t>0$, this amounts to introducing a time integral in (4) as [10]

$$
\mathcal{M}_{\mathcal{L}_{p}}:=N^{\frac{2}{n}}\left(\int_{0}^{T} \frac{1}{\Delta t}\left(\int_{\Omega} \operatorname{det}(\mathcal{M})^{\frac{p}{2 p+n}} \mathrm{~d} x\right) \mathrm{d} t\right)^{-\frac{2}{n}} \operatorname{det}(\mathcal{M})^{\frac{-1}{2 p+n}} \mathcal{M} .
$$

In the time-dependent case, it should be noted that $N$ is now a space-time complexity, given by integrating (2) in time. The discrete analogue is the number of mesh vertices summed over all timesteps.

\subsection{Combining Metric Information}

Suppose now that we have a finite number of Riemannian metrics, each of which captures some aspect of the PDE solution, or an error estimate thereof. For instance, they might be Hessians of different components of a vector solution field.

The most straightforward way to combine these metrics is to take the average (on an entry-by-entry basis). That this yields a metric follows from the definition of positive-definiteness. Alternatively, metrics can be combined using intersection (also known as superposition). Unlike averaging, this combination method always yields meshes with more elements than those which would arise from the constituent metrics. For details on metric intersection, see pp.3778-3779 of [9].

An investigation into the properties of metric intersection and averaging was made on pp.131-138 of [27]. In the context of unsteady adaptation applied to advection problems, metric intersection was found to deal better with shocks. However, metric averaging was found to be more effective at resolving sharp angles and small scale features.

It is necessary to scale metrics (using the methods in Subsection 2.2, for example) before combining them. In the case where two metrics have been constructed from the Hessians of different fields, for example, there is no guarantee that these second derivative matrices are of the same order of magnitude. Normalising before combination means that the Hessians may be averaged or superimposed in a meaningful way. This comes at the cost of the metrics being constrained by the target complexity.

\subsection{Metric Gradation}

Metric gradation techniques are usually applied to metric fields to ensure that the sizes they prescribe at neighbouring vertices do not vary by more than a specified threshold, taken here as $\beta=1.4$. That is, the ratio of the prescribed sizes is bounded from above by $\beta$. This has the effect that meshes resulting from the metric-based mesh adaptation routine do not have sudden changes in resolution, which could act as artificial internal boundaries in the flow. For details on the metric gradation algorithm used, see [25]. 


\subsection{Software}

Anisotropic mesh adaptation is achieved in Firedrake using Pragmatic [28]. This C++ library takes as input a triangular or tetrahedral mesh and a metric field over it and returns a mesh which is adapted to the metric. The adapted mesh is derived from the input one through a series of mesh modifications (edge splits, collapses and swaps and vertex smoothing in the 2D case) that optimise the lengths of the edges as well as a quality functional. More detail can be found in [29, 30, 31]. Pragmatic and Firedrake are interfaced through PETSc [28, 32, 33, 34]. High-level error estimate information is passed to Pragmatic using Riemannian metric fields constructed in Firedrake from FEM solutions of the PDE and its adjoint.

\section{Goal-Oriented Error Estimation}

\subsection{Tracer Transport Problem}

This paper focuses on the application of goal-oriented error estimation and mesh adaptation to tracer transport problems. The prognostic variable, $c$, is the concentration of a passive tracer immersed in a fluid with velocity, $\mathbf{u}$, and SPD diffusivity tensor, $\underline{\boldsymbol{D}}$. For a source term $S$, consider the steady-state tracer transport problem,

$$
\left\{\begin{array}{rlrl}
\mathbf{u} \cdot \nabla c-\nabla \cdot(\underline{\boldsymbol{D}} \nabla c) & =S & & \text { in } \Omega \\
\underline{\boldsymbol{D}} \nabla c \cdot \widehat{\mathbf{n}} & =g_{N} & & \text { on } \partial \Omega_{N} \\
c & =g_{D} & & \text { on } \partial \Omega_{D}
\end{array} .\right.
$$

The domain boundary, $\partial \Omega$, is assumed to be piecewise smooth, decomposed into a disjoint union of open boundary, Dirichlet and Neumann components as $\partial \Omega=\partial \Omega_{\text {open }} \cup \partial \Omega_{D} \cup \partial \Omega_{N}$.

Let $V$ be a function space containing the exact solution, $c$, of (6). For a Continuous Galerkin (CG) discretisation, we have a weak formulation

$$
a(c, v)=L(v), \quad \forall v \in V
$$

comprised of a bilinear form $a: V \times V \rightarrow \mathbb{R}$ and linear form $L: V \rightarrow \mathbb{R}$,

$$
\begin{aligned}
a(c, v) & :=\langle\mathbf{u} \cdot \nabla c, v\rangle+\langle\underline{\boldsymbol{D}} \nabla c, \nabla v\rangle-\left\langle\underline{\boldsymbol{D}} \nabla c \cdot \widehat{\mathbf{n}}_{K}, v\right\rangle_{\partial \Omega \backslash \partial \Omega_{N}}, \\
L(v) & :=\langle S, v\rangle+\left\langle g_{N}, v\right\rangle_{\partial \Omega_{N}} .
\end{aligned}
$$

Here $\langle\cdot, \cdot\rangle$ denotes the usual $\mathcal{L}_{2}$ inner product on $\Omega$. For other spaces $S$, we use the notation $\langle\cdot, \cdot\rangle_{S}$. In addition, denote $\|\cdot\|:=\langle\cdot, \cdot\rangle$ and $\|\cdot\|_{S}:=\langle\cdot, \cdot\rangle_{S}$.

Integration by parts has been applied in deriving (8), to apply Neumann boundary conditions and reduce the order of derivatives. The weak formulation is also important for the construction of goal-oriented error estimators in Subsection 3.3 .

Equation (6) gives rise to the strong residual,

$$
\Psi(c):=S-\mathbf{u} \cdot \nabla c+\nabla \cdot(\underline{\boldsymbol{D}} \nabla c) .
$$

Similarly, equation (7) gives rise to the weak residual,

$$
\rho(c, v):=L(v)-a(c, v) .
$$

For a finite dimensional subspace $V_{h} \subset V$, the Galerkin approximation,

$$
\rho\left(c_{h}, v\right)=L(v)-a\left(c_{h}, v\right)=0, \quad \forall v \in V_{h},
$$

admits a finite element solution, $c_{h} \in V_{h}$. In this work we use a CG method with polynomial degree 1 , i.e. $V_{h}=\mathbb{P} 1$. That is, the tracer concentration is assumed to be piecewise linear on each mesh element, with continuity imposed across inter-element boundaries.

Stabilisation terms are usually added to (7) in order to control under- and over-shoots that are typical in advectiondominated problems. Streamline Upwind Petrov-Galerkin (SUPG) stabilisation is applied, implying an additional term $\langle\Psi(c), \tau \mathbf{u} \cdot \nabla v\rangle$, where $\tau>0$ is a stabilisation parameter.

In this work, we make the standard choice of stabilisation parameter [35],

$$
\left.\tau\right|_{K}:=\frac{h_{K}}{2\|\mathbf{u}\|_{2}} \min \left(1, \frac{\mathrm{Pe}_{h}}{3}\right), \quad \text { where } \quad \mathrm{Pe}_{h}:=\frac{h_{K}\|\mathbf{u}\|_{2}}{2 D}
$$


is the mesh Péclet number and $h_{K}$ is a cell size measure for element $K$. A typical choice of cell size is the cell diameter, i.e. the circumradius for triangular and tetrahedral elements. For isotropic elements, this is a suitable choice and it scales with element volume. However, it can give very large values for highly anisotropic elements. In the triangular case, for example, the area of a highly anisotropic element is much smaller than an element with all edges the same length as the anisotropic element's longest edge. For anisotropic elements, a more suitable measure of cell size is that advocated in [36]. If $\underline{J}_{K}$ is the Jacobian of the map from a reference element, $\widehat{K}$, to some mesh element $K \in \mathcal{H}$, then cell size is may be defined as the minimum eigenvalue of the SPD part of a polar decomposition, $\underline{\boldsymbol{J}}_{K}=\underline{\boldsymbol{B}}_{K} \underline{\boldsymbol{Z}}_{K}$. For further details on the SUPG stabilisation strategy, see [35, 36].

The linear system obtained after discretisation and stabilisation is solved using a direct method [37, 38].

\subsection{Adjoint Tracer Transport Problem}

Given the strong residual (9), the 'forward equation' (in this case the advection-diffusion equation) may be written in 'residual form' as

$$
\Psi(c)=0 .
$$

In addition, we introduce a scalar diagnostic which is held to be important for some use case - the QoI, $J: V \rightarrow \mathbb{R}$. The adjoint equation associated with (13) and $J$ is given by

$$
\left(\frac{\partial \Psi}{\partial c}(c)\right)^{T} c^{*}=\frac{\partial J^{T}}{\partial c}, \quad \text { where } \quad c^{*} \in V
$$

is the adjoint solution. Due to the linearity of the advection-diffusion equation, the derivative term on the LHS of (14) is actually independent of the forward solution. Therefore, the adjoint equation may be solved independently of the forward equation, provided the $\mathrm{QoI}$ is also linear. This is not true for nonlinear problems.

The derivation of an adjoint problem (14) involves making a choice: should the equation be differentiated and then discretised (i.e. the continuous adjoint approach), or discretised and then differentiated (i.e. the discrete adjoint approach)? In this work, we make use of the discrete adjoint functionality made available in Firedrake by the dolfinadjoint library [39]. Doing so avoids tedious and error-prone manual calculations and ensures continuity of functional gradients [40].

\subsection{Estimating QoI Error}

Due to the goal-oriented error analysis of [2], we have the classical result,

$$
J(c)-J\left(c_{h}\right)=\rho\left(c_{h}, c^{*}-c_{h}^{*}\right)+R,
$$

where the remainder term, $R$, is quadratic in the forward and adjoint errors, $e=c-c_{h}$ and $e^{*}=c^{*}-c_{h}^{*}$. Equation (DWR may be used to define goal-oriented error estimators, as outlined in Subsections 3.4 and 3.5

A 'second-order' result may also be derived [2]; error estimates deduced from this result are applied to anisotropic goal-oriented mesh adaptation for tracer transport problems in [13]. In this paper, however, we focus on the first order result (DWR), termed the dual-weighted residual. Note that the remainder term vanishes in the case where the prognostic equation is linear and the QoI is quadratic [2]. By linearity of [6], (DWR) gives an exact representation of the QoI error, provided that the QoI is quadratic.

The error result stated above is global, in the sense that it quantifies QoI error incurred over the whole domain. Its use within a mesh adaptation routine requires localisation, so that we may deduce the error contributions incurred in different parts of the domain. More specifically, we seek to deduce the error incurred in each element, $K \in \mathcal{H}$. Clearly, the weak residual may be decomposed as a sum of contributions from each mesh element. Therefore, element-wise error indicators, $\eta_{K}$, may be extracted from (DWR) as

$$
\eta_{K}:=\left|\rho\left(c_{h}, c^{*}-c_{h}^{*}\right)\right|_{K} \mid, \quad K \in \mathcal{H} .
$$

Summing over all elements yields a global error estimator. This provides an upper bound for the QoI error, by the triangle inequality.

Recall that integration by parts was applied over the whole domain when deriving the variational formulation (8). Error indicators of the form (15) may be decomposed using a second integration by parts. Now the domain of integration is each mesh element, since we seek element-wise error indicators. Inter-element flux terms are introduced, as well as boundary terms,

$$
\left.\rho\left(c_{h}, e^{*}\right)\right|_{K}=\left\langle\Psi\left(c_{h}\right), e^{*}\right\rangle_{K}+\left\langle\psi^{N}\left(c_{h}\right), e^{*}\right\rangle_{\partial K \cap \partial \Omega_{N}}+\left\langle\psi^{\text {flux }}\left(c_{h}\right), e^{*}\right\rangle_{\partial K \backslash \partial \Omega},
$$


where $\psi^{N}$ is the residual of the Neumann boundary conditions and $\psi^{\text {flux }}$ corresponds to flux terms between elements. The magnitude of the first term tells us how well the PDE is solved across the domain. The second term conveys how well the Neumann conditions have been satisfied. The third term is related to the smoothness of the solution field across inter-element boundaries. For CG methods (such as used here) the flux terms arise purely due to the integration by parts. Discontinuous discretisations contribute additional flux terms, by construction (for example, see [18]).

For the stabilised version of the CG formulation given in (7), integrating by parts yields goal-oriented error indicators

$$
\begin{aligned}
\left.\rho\left(c_{h}, e^{*}\right)\right|_{K} & =\left\langle\Psi\left(c_{h}\right), e^{*}\right\rangle_{K} \\
& +\left\langle\underline{\boldsymbol{D}} \nabla c_{h} \cdot \widehat{\mathbf{n}}_{K}-g_{N}, e^{*}\right\rangle_{\partial K \cap \partial \Omega_{N}} \\
& +\left\langle\underline{\boldsymbol{D}} \nabla c_{h} \cdot \widehat{\mathbf{n}}_{K}, e^{*}\right\rangle_{\partial K \backslash \partial \Omega} \\
& +\left\langle\Psi\left(c_{h}\right), \tau \mathbf{u} \cdot \nabla e^{*}\right\rangle_{K} .
\end{aligned}
$$

That is, the terms on the RHS correspond to those given in (16), along with an additional stabilisation error term. When summed over all elements of the mesh, the inner product over $\partial K \backslash \partial \Omega$ in $(17)$ corresponds to a flux jump term. Observe that if $c_{h}$ is replaced with the (assumed smooth) exact solution of (6) then this estimator vanishes, as we might hope.

\section{4 'Isotropic DWR Metric'}

The simplest way to create a metric from a scalar error indicator such as $[15]$ is to use it to scale an identity matrix. Doing so yields a $\mathbb{P} 0$ tensor field. In particular, it takes the form of a diagonal matrix with positive diagonal entries in every mesh element. Therefore, the field is SPD and hence a valid metric. However, the metric-based mesh adaptation formulation used in this paper requires the metric to be defined in $\mathbb{P} 1$ space. Therefore, an additional projection step is required. Given an appropriate projection operator $\Pi_{1}: \mathbb{P} 0 \rightarrow \mathbb{P} 1$, an isotropic metric may be defined as

$$
\mathcal{M}^{\mathrm{DWR}}:=\Pi_{1}\left(\sum_{K \in \mathcal{H}} \eta_{K} \mathbb{1}_{K}\right) \underline{\boldsymbol{I}}_{n},
$$

where $\underline{\boldsymbol{I}}_{n}$ is the identity matrix in $n$ dimensions. In this work, nodewise values are generated by volume-averaging $\eta_{K}$ over adjacent elements [41].

Metrics of the form (18) allow control of element sizes under mesh adaptation, but not their shape or orientation. Therefore, we refer to (18) as the isotropic DWR metric. Metrics should always be normalised (for example, using the methods outlined in Subsection 2.2, in order to make them relevant to the problem at hand.

\subsection{Anisotropic Goal-Oriented Error Estimation}

As discussed in Section 1, the literature contains a number of approaches for extending the goal-oriented error estimation framework to obtain anisotropic meshes. Some notable contributions are applied to the tracer transport modelling problem in the following.

\subsection{1 'Anisotropic DWR Metric'}

The first anisotropic metric we consider follows the work of [3, 5]. Like [18], this approach is based on the a posteriori error result (DWR) and uses an element-wise formulation. Where in Section 2 Hessians are constructed on a vertex-wise basis, let $\underline{\boldsymbol{H}}_{K}$ denote the Hessian of the forward solution constructed on element $K$. It also admits an orthogonal eigen-decomposition with eigenvector matrix $\underline{\boldsymbol{V}_{\boldsymbol{K}}}$ and eigenvalues $\left\{\lambda_{K, i}\right\}_{i=1}^{n}$. The stretching factors

$$
s_{K, i}:=\left(\prod_{j=1}^{n}\left|\lambda_{K, j}\right|\right)^{-\frac{1}{n}}\left|\lambda_{K, i}\right|, \quad i=1, \ldots, n
$$

are element shape deformations in the directions defined by the eigenvectors. Without loss of generality, assume $\left|\lambda_{K, 1}\right| \geq \cdots \geq\left|\lambda_{K, n}\right|>0$, so that $s_{K, 1} \geq \cdots \geq s_{K, n}>0$. In the 2D case, $s_{K, 1}^{2}=\left|\lambda_{K, 1} / \lambda_{K, 2}\right|$ and $s_{K, 2}^{2}=$ $\left|\lambda_{K, 2} / \lambda_{K, 1}\right|$ are the maximal and minimal anisotropy ratios in the element-wise Hessian.

Whilst the normalisation strategy used for this metric is also focused on metric complexity, it differs from the approach described in Subsection 2.2 in that it incorporates error indicators. As described in [5], an element volume $|\widetilde{K}|$ is sought such that the metric complexity is smaller than some target complexity, $N$. Within the element-wise framework, this optimisation problem is shown in [42] to be solved by the choice

$$
|\tilde{K}|:=\frac{|K|}{N}\left(\sum_{K \in \mathcal{H}} \eta_{K}^{\frac{1}{\alpha+1}}\right) \eta_{K}^{-\frac{1}{\alpha+1}},
$$


where $|K|$ is the volume of element $K$. The tunable parameter $\alpha \geq 1$ may be interpreted as akin to the normalisation degree in $\mathcal{L}_{p}$ normalisation.

An anisotropic metric is constructed from 20, by reassembling the element-wise Hessian with a modified eigenvalue matrix. Again, a vertex-based metric is obtained by projection as

$$
\mathcal{M}^{\mathrm{ADWR}}:=\Pi_{1}\left(\sum_{K \in \mathcal{H}} \frac{|\widehat{K}|}{|\widetilde{K}|} \underline{\boldsymbol{V}}_{K} \operatorname{diag}\left(s_{K, 1}, \ldots, s_{K, n}\right) \underline{\boldsymbol{V}}_{K}^{T} \mathbb{1}_{K}\right),
$$

where $|\widehat{K}|$ is the volume of the reference element, $\widehat{K}$.

\subsection{2 'Weighted Hessian Metric'}

The second anisotropic metric we consider is again derived from the dual-weighted residual. Like the approach described above, this metric - first presented in [14] - also makes use of the Hessian to incorporate anisotropy. This is done through the following reinterpretation of (DWR):

$$
J(c)-J\left(c_{h}\right) \approx\left\langle\Psi\left(c_{h}\right), c^{*}-\Pi_{h} c^{*}\right\rangle \leq\left\|\Psi\left(c_{h}\right)\right\|\left\|c^{*}-\Pi_{h} c^{*}\right\|,
$$

where the inequality follows by Cauchy-Schwarz. That is, the interface term is neglected and the approximate adjoint solution is understood as a projection of the true adjoint solution into the finite element space, $V_{h}$. Using the relation (1) between interpolation error and the Hessian, an anisotropic metric may be constructed from (22) by weighting the Hessian of the adjoint solution with the projection of the forward residual into $\mathbb{P} 1$ space:

$$
\mathcal{M}^{\mathrm{WH}}:=\Pi_{1}\left(\sum_{K \in \mathcal{H}}\left\|\Psi_{h}\left(c_{h}\right)\right\|_{K} \mathbb{1}_{K}\right)\left|\underline{\boldsymbol{H}}\left(c_{h}^{*}\right)\right|,
$$

In practice, a $\mathbb{P} 1$ Hessian is recovered for the approximate adjoint solution. Henceforth, 23] is termed the 'weighted Hessian' (WH) anisotropic metric.

\section{'Weighted Gradient Metric'}

For the purposes of defining the final metric, use $\Psi_{h}(\cdot)$ to denote the strong residual in finite element space, as opposed to the 'true' strong residual, $\Psi(\cdot)$. Instead of being based on (DWR), this metric uses an alternative error result derived in [15]:

$$
J(c)-J\left(c_{h}\right)=\left\langle\left(\Psi_{h}-\Psi\right)(c), c^{*}\right\rangle+\widetilde{R},
$$

where $\widetilde{R}$ is a remainder term which now involves interpolation errors on $V_{h}$ and the adjoint error, $e^{*}$. As with the WH metric, we begin by noting that (24) contains what may be interpreted as an interpolation error. However, where before this was an interpolation error in the adjoint solution, here it is an interpolation error in the PDE itself.

Suppose the PDE can be written in conservative form as

$$
\Psi(c)=\nabla \cdot \mathcal{F}(c)=0,
$$

where $\mathcal{F}=\left(\mathcal{F}_{1}, \ldots, \mathcal{F}_{n}\right)$. Integrating 24 by parts yields

$$
J(c)-J\left(c_{h}\right) \approx\left\langle\left(\mathcal{F}-\Pi_{h} \mathcal{F}\right)(c), \nabla c^{*}\right\rangle-\left\langle\left(\widehat{\mathbf{n}} \cdot\left(\overline{\mathcal{F}}-\Pi_{h} \overline{\mathcal{F}}\right)(c), c^{*}\right\rangle_{\partial \Omega},\right.
$$

where $\overline{\mathcal{F}}$ is a residual for the boundary conditions. That is, $\Psi(c)=0$ if and only if $c$ solves the PDE on the domain interior and $\overline{\mathcal{F}}(c)=0$ if and only if the boundary conditions are satisfied exactly. Application of the Cauchy-Schwarz and triangle inequalities yields the bound

$$
\begin{aligned}
\left|J(c)-J\left(c_{h}\right)\right| \lesssim & \sum_{i=1}^{n}\left\|\left(\mathcal{F}_{i}-\Pi_{h} \mathcal{F}_{i}\right)(c)\right\|\left\|\frac{\partial c^{*}}{\partial x_{i}}\right\| \\
& +\left\|\widehat{\mathbf{n}} \cdot\left(\overline{\mathcal{F}}-\Pi_{h} \overline{\mathcal{F}}\right)(c)\right\|_{\partial \Omega}\left\|c^{*}\right\|_{\partial \Omega} .
\end{aligned}
$$

Approximate bound 27 involves interpolation errors in both $\overline{\mathcal{F}}$ and the components $\mathcal{F}_{i}$. In [15], it is argued that interpolation error bounds of the form (1) may be applied on a component-by-component basis, in order to construct anisotropic metrics. Hessians are weighted by the adjoint or components of its gradient, as appropriate:

$$
\underline{\boldsymbol{H}}^{\text {volume }}:=\sum_{i=1}^{n}\left|\underline{\boldsymbol{H}}\left(\mathcal{F}_{i}(c)\right)\right|\left|\frac{\partial c^{*}}{\partial x_{i}}\right|, \quad \underline{\boldsymbol{H}}^{\text {surface }}:=\left|c^{*}\right||\underline{\boldsymbol{H}}(\widehat{\mathbf{n}} \cdot \overline{\mathcal{F}}(c))| .
$$


The resulting metrics are then intersected on the boundary, giving

$$
\mathcal{M}^{\mathrm{WG}}:= \begin{cases}\left|\underline{\boldsymbol{H}}^{\text {volume }}(\mathbf{x})\right| \cap\left|\underline{\boldsymbol{H}}^{\text {surface }}(\mathbf{x})\right| & \mathbf{x} \in \partial \Omega \\ \left|\underline{\boldsymbol{H}}^{\text {volume }}(\mathbf{x})\right| & \mathbf{x} \in \Omega\end{cases}
$$

Note that the error result 24) which this metric is based upon is a priori, whilst all previously considered metrics are a posteriori. We make this classification by following [15] in interpreting the a posteriori standpoint as assuming knowledge of errors incurred on an existing mesh and the a priori standpoint as assuming knowledge of the PDE solution. That is, the a posteriori metrics involve residuals evaluated on the current mesh, whereas the a priori metric 29] involves exact solutions for the forward and adjoint PDEs, making no reference to finite element solutions. As in the WH approach, these exact values are approximated by finite element solutions in practice, replacing instances of $c$ and $c^{*}$ with $c_{h}$ and $c_{h}^{*}$, respectively. The difference in classification has to do with the derivation of the methods, rather than implementation details. Henceforth, 29 is referred to as the 'weighted gradient' (WG) anisotropic metric, due to the gradient term in the domain interior contribution.

\subsubsection{Modifications}

Whilst the anisotropic methods considered are inspired by the work of [3, 14, 15], there are some notable differences between the original implementations and those used in this work, as outlined in the following.

The construction of the WG metric (29) requires the prognostic equation (6) to be in conservative form. Assuming the velocity field to be divergence-free, the tracer transport equation may be expressed in terms of a potential functional $\mathcal{F}$ as

$$
\nabla \cdot \mathcal{F}(c)=S, \quad \mathcal{F}(c)=\mathbf{u} c-\underline{D} \nabla c .
$$

However, the source term, $S$, requires special treatment. In this work we construct the metric contribution in the domain interior by the sum:

$$
\mathcal{M}_{S}^{\mathrm{WG}}:=\left|\underline{\boldsymbol{H}}\left(\mathcal{F}_{1}(c)\right)\right|\left|\frac{\partial c^{*}}{\partial x}\right|+\left|\underline{\boldsymbol{H}}\left(\mathcal{F}_{2}(c)\right)\right|\left|\frac{\partial c^{*}}{\partial y}\right|+|\underline{\boldsymbol{H}}(S)|\left|c^{*}\right| .
$$

The first two terms on the RHS correspond to (28). For the boundary term, recall that we consider three types of boundary segment: Neumann, Dirichlet and open. Neumann conditions are usually weakly imposed in finite element methods, giving

$$
\left.\widehat{\mathbf{n}} \cdot \overline{\mathcal{F}}(c)\right|_{\partial \Omega_{N}}=g_{N}-\underline{\boldsymbol{D}} \nabla c \cdot \widehat{\mathbf{n}} .
$$

As described above, (32) conveys the extent to which the Neumann conditions are enforced. For CG methods, Dirichlet conditions are usually strongly imposed, contributing zero error. Boundary integrals over open boundaries are cancelled by the integration by parts. Therefore, these segments do not contribute to the surface metric.

It is worth remarking that, whilst stabilisation errors have been accounted for by the DWR indicators used in both isotropic and anisotropic metric constructions, they are not considered in the WG method, because it arises from an a priori error result, which makes no mention of the discretisation. For the WH approach, we can account for stabilisation by making use of the fact that SUPG is a Petrov-Galerkin method. That is, we use the construction

$$
\mathcal{M}_{\mathrm{SUPG}}^{\mathrm{WH}}:=\Pi_{1}\left(\sum_{K \in \mathcal{H}}\left\|\Psi_{h}\left(c_{h}\right)\right\|_{K} \mathbb{1}_{K}\right)\left|\underline{\boldsymbol{H}}\left(c_{h}^{*}+\tau \mathbf{u} \cdot \nabla c_{h}^{*}\right)\right| .
$$

As with the isotropic metric, the WG $(31)$ and WH $(33)$ metrics are normalised using $\mathcal{L}_{p}$ normalisation, as in (4). Unless otherwise stated, the normalisation order $p=1$ is assumed, since it admits multi-scale meshes, whilst being able to capture sharp features, such as point sources. The anisotropic DWR method has inbuilt normalisation, for which we find the parameter value $\alpha=2$ to be effective in practice.

\subsection{Error Estimate Evaluation}

An important aspect of goal-oriented error estimation is the treatment of the (clearly unknown) adjoint error term, $e^{*}$, which appears in (DWR). One approach is to approximate $c^{*}$ by solving the adjoint problem again in a globally enriched space. Enrichment can mean incrementing the polynomial degree ( $p$-refinement), subdividing elements $(h$-refinement) or a combination thereof. In any case, the enrichment process leads to a heightened DoF count. In [13], an enriched adjoint solution was obtained using both $h$ - and $p$-refinement. This was found to be effective for goal-oriented error estimation and mesh adaptation for the same steady-state advection diffusion test case as considered in the following 
section. However, it comes with a far higher computational cost than solving the forward problem in the base space, which is clearly undesirable.

In this work, we instead utilise the 'difference quotient' approach described in [2]. No enrichment is required; instead, the error indicator is modified and evaluated using only solution fields from the base space. Combining flux and boundary terms as $\psi(\cdot):=\psi^{\partial}(\cdot)+\psi^{\text {flux }}(\cdot)$, application of the Cauchy-Schwarz inequality yields the upper bound

$$
\left|\rho\left(c_{h}, e^{*}\right)\right|_{K} \mid \leq\left(\left\|\Psi\left(c_{h}\right)\right\|_{K}+h_{K}^{-\frac{1}{2}}\left\|\psi\left(c_{h}\right)\right\|_{\partial K}\right) \underbrace{\left(\left\|e^{*}\right\|_{K}+h_{K}^{\frac{1}{2}}\left\|e^{*}\right\|_{\partial K}\right)}_{\omega_{K}} .
$$

Again, we may interpret $c_{h}^{*}$ as the projection of $c^{*}$ into the finite element space. Thus, for some interpolation constant, $C>0$, it satisfies [43]

$$
\omega_{K}=\left\|c^{*}-\Pi_{h} c^{*}\right\|_{K}+h_{K}^{\frac{1}{2}}\left\|c^{*}-\Pi_{h} c^{*}\right\|_{\partial K} \leq C h_{K}^{2}\left\|\nabla^{2} c^{*}\right\|_{K} \cdot
$$

We follow [2] in approximating the Laplacian $\nabla^{2} c^{*}$ by that the Laplacian $\nabla^{2} c_{h}^{*}$, as obtained using a recovery technique. In practice, it is difficult to determine $C$, meaning that (34) cannot be used for the evaluation of global error estimates. Nonetheless, its localisation to individual elements can be readily used in a mesh adaptation algorithm, where overall scaling constants are irrelevant. One possible localisation reads

$$
\eta_{K}^{\mathrm{DQ}}:=\left(\left\|\Psi\left(c_{h}\right)\right\|_{K}+h_{K}^{-\frac{1}{2}}\left\|\psi\left(c_{h}\right)\right\|_{\partial K}\right)\left\|\nabla^{2} c_{h}^{*}\right\|_{\partial K}, \quad K \in \mathcal{H}
$$

Similarly as with the WH metric in 33 , an error indicator which accounts for SUPG stabilisation may be obtained as

$$
\left(\eta_{\mathrm{SUPG}}^{\mathrm{DQ}}\right)_{K}:=\left(\left\|\Psi\left(c_{h}\right)\right\|_{K}+h_{K}^{-\frac{1}{2}}\left\|\psi\left(c_{h}\right)\right\|_{\partial K}\right)\left\|\nabla^{2}\left(c_{h}^{*}+\tau \mathbf{u} \cdot \nabla c_{h}^{*}\right)\right\|_{\partial K}
$$

For the WH metric, the adjoint error is already approximated using the Hessian. As such, the above discussion is not of concern. For WG, we follow the authors of [15] in approximating gradients in the exact adjoint solution by gradients in the numerical approximation thereof. Therefore, the difference quotient formulation is only required for the isotropic and anisotropic DWR metrics.

\section{Steady-State Goal-Oriented Mesh Adaptation}

First, consider the time-independent case.

\subsection{Mesh Adaptation Approach}

Given an initial mesh $\mathcal{H}_{0}$ and a desired metric complexity $N>0$, we construct a sequence of meshes $\left\{\mathcal{H}_{i}\right\}_{i \in \mathbb{N}}$ by iteratively solving the forward problem (6) and its (discrete) adjoint and constructing an appropriate metric from their solution fields. Algorithm 1 provides a workflow representation.

Given target metric complexity $N>0$;

Given initial mesh $\mathcal{H}_{0}$;

Set $i:=0$;

while not converged do

Solve forward problem on $\mathcal{H}_{i}$;

Evaluate the QoI and check for its convergence;

Solve adjoint problem on $\mathcal{H}_{i}$;

Construct metric $\mathcal{M}_{i}$, normalising for target complexity $N$;

Apply metric gradation to $\mathcal{M}_{i}$;

Adapt the mesh under $\mathcal{M}_{i}$ to obtain $\mathcal{H}_{i+1}$;

Increment $i$;

end

Algorithm 1: Mesh adaptation routine for time-independent problems.

Convergence is attained when the relative change in one of the (a) QoI value, (b) mesh element count, or (c) DWR error estimator (if evaluated) falls below some tolerance, taken here as $0.5 \%$. A maximum iteration count of 35 is also imposed. For a relatively isotropic $\mathcal{H}_{0}$, we find that at least three mesh iterations are required in order to introduce anisotropy into meshes produced by Algorithm 1 . Therefore, three iterations are performed before the convergence criteria are checked. 


\subsection{Point Discharge Test Case}

Numerical experiments in this subsection focus on the 'Point Discharge with Diffusion' test case from the TELEMAC$2 \mathrm{D}$ validation document version 7.0 [24]. This steady-state advection-diffusion problem is defined in a rectangular domain, $\Omega=[0,50] \times[0,10] \mathrm{m}^{2}$, with prescribed uniform flow velocity, $\mathbf{u}=\left(u_{1}, u_{2}\right)=(1,0) \mathrm{m} \mathrm{s}^{-1}$, and isotropic diffusivity tensor, $\underline{\boldsymbol{D}}=D \underline{\boldsymbol{I}}$, where $D=0.1 \mathrm{~m}^{2} \mathrm{~s}^{-1}$. A tracer is injected into the flow at $\mathrm{x}_{0} \in \Omega$, and specified in the continuous problem via a Dirac delta function, i.e. spatially a point source. An inflow (Dirichlet) condition, $c=0$, is imposed at the left-hand boundary, along with Neumann conditions on the North and South boundaries and an open right-hand boundary.

The only difference between the test case presented here and the original one in [24] is that we follow [13] in locating the point source at $\mathbf{x}_{0}=(2,5)$, rather than $\mathbf{x}_{0}=(1,5)$. We consider the same initial mesh as in [24], comprised of a uniform grid of 4,000 isosceles right-angled triangles.

\subsubsection{Calibration}

An advantage of this test case is that it has an analytical solution, [24]

$$
c_{\text {analytical }}(\mathbf{x}):=\frac{q}{2 \pi D} \exp \left(\frac{u_{1} x}{2 D}\right) K_{0}\left(\frac{u_{1}\left\|\mathbf{x}-\mathbf{x}_{0}\right\|_{2}}{2 D}\right),
$$

where $q$ denotes the inflow discharge at the source and $K_{0}$ is the zeroth order modified Bessel function of the second kind. Following [24], we set $q=100 \mathrm{gl}^{-1}$.

Note that $K_{0}$ blows up at zero, in correspondence with 38 becoming infinite at $\mathbf{x}_{0}$. Therefore, we approximate the $\ell_{2}$ norm in 38 using

$$
d(\mathbf{x}):=\max \left(\left\|\mathbf{x}-\mathbf{x}_{0}\right\|_{2}, r\right),
$$

which has the effect of introducing a plateau in the region $B_{r}\left(\mathbf{x}_{0}\right)$, for some $r>0$. Here we use the notation

$$
B_{\epsilon}(\mathbf{y}):=\left\{\mathbf{x} \in \Omega \mid\|\mathbf{x}-\mathbf{y}\|_{2} \leq \epsilon\right\},
$$

for the ball of radius $\epsilon>0$, centred at $\mathbf{y} \in \Omega$.

Point sources cannot generally be easily represented in numerical models. Therefore, we use the radius $r$ again when representing the source. In [13], the source is represented by an indicator function of narrow radius, which effectively replaces the point source with a pipe of radius $r$. In this work, we instead adopt a Gaussian approximation,

$$
S(\mathbf{x}):=q \exp \left(-\frac{\left\|\mathbf{x}-\mathbf{x}_{0}\right\|_{2}^{2}}{r^{2}}\right)
$$

meaning that $S$ is smooth, rather than discontinuous.

In [13], a calibrated value of $r$ was established by trial and error. Here we take a more rigorous calibration approach using gradient-based optimisation. The aim of this optimisation is to minimise the functional

$$
J_{\text {calibration }}(c):=\int_{\Omega \backslash B_{r}\left(\mathbf{x}_{0}\right)}\left(c-c_{\text {analytical }}\right)^{2} \mathrm{~d} x,
$$

subject to the constraint that $r$ remains strictly positive. The $c$ used to evaluate (42) is obtained by solving the tracer transport equation with some value of $r$. Due to the plateau enforced by (39), we do not seek to match the analytical solution within the source region.

Running the optimisation using L-BFGS-B [44] on a fine uniform mesh with 1,024,000 elements gives the calibrated radius $r=5.606535 \mathrm{~cm}$. The resulting finite element solution on this fine mesh is compared against the analytical solution in Figure 1. An initial qualitative observation is that the stabilised FEM approximation on the fine uniform mesh provides an excellent approximation to the analytical solution. It appears that the only region where there is a noticeable difference between the analytical and finite element solutions is near the boundary for $x \in[30,50]$. This is in agreement with what may be observed in the TELEMAC-2D solution given in [24].

\subsubsection{Quantities of Interest}

The functional (42) used for calibration is nonlinear and does not have a simple dependence on the control parameter, $r$. For the goal-oriented mesh adaptation experiments in the following, linear functionals of the form

$$
J_{i}(c):=\int_{\Omega} \mathbb{1}_{R_{i}} c \mathrm{~d} x=\int_{R_{i}} c \mathrm{~d} x, \quad R_{i}=B_{\frac{1}{2}}\left(\mathbf{x}_{i}\right)
$$




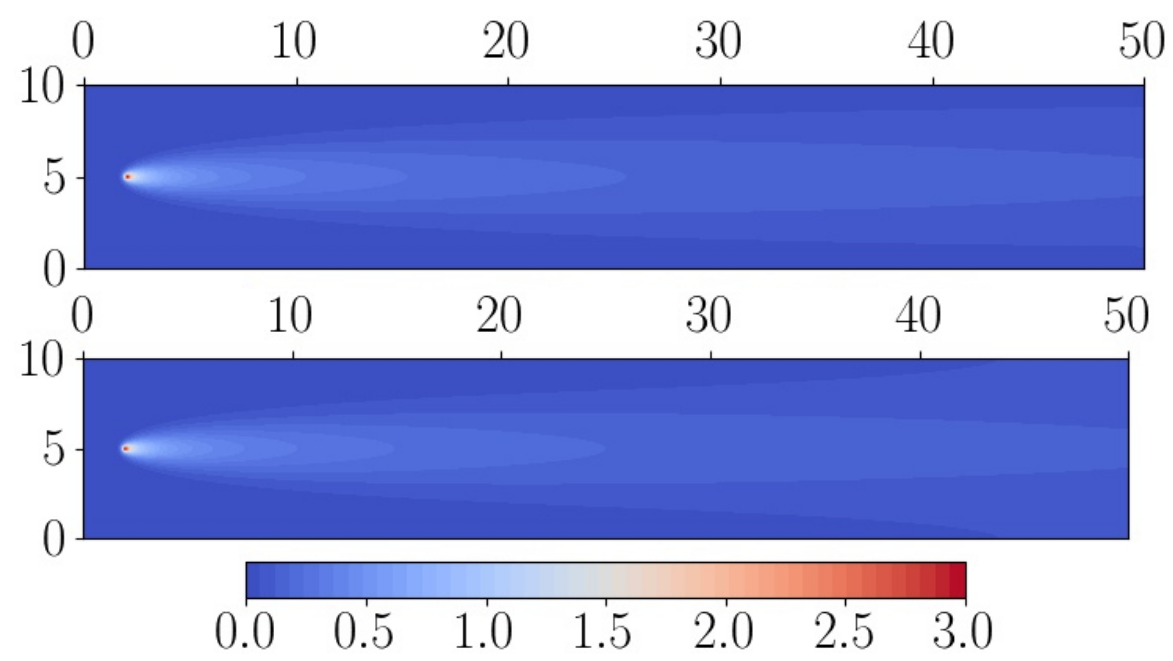

(a) Analytical solution.

Figure 1: Analytical and finite element solutions for the 'Point Discharge with Diffusion' test case, with calibrated radius $5.606535 \mathrm{~cm}$ for the source term. Each field is presented in $\mathbb{P} 1$ space on a uniform mesh with 1,024,000 elements and 513,921 vertices.

are used. Evaluating (43) amounts to measuring the tracer concentration in a 'receiver region' centred at $\mathbf{x}_{i} \in \Omega$.

As in the similar steady-state advection-diffusion test case examined in [14], we consider two scenarios: one where the receiver is directly downstream from the source and one where it is offset to one side of the channel. We choose $\mathbf{x}_{1}=(20,5)$ in the former instance and $\mathbf{x}_{2}=(20,7.5)$ in the latter. Goal-oriented mesh adaptation seeks a mesh which allows an accurate approximation of the tracer concentration integrated over the receiver region.

For a related application in environmental science, consider the inlet and outlet pipes of a desalination plant. The inlet pipe brings sea water into the plant, from region $R_{i}$. Salt is extracted from this water and the remaining salt residues are released back into the ocean through the outlet pipe, depositing at $\mathbf{x}_{0}$. An undesirable - but entirely possible - situation is where a significant quantity of salt from the outlet pipe is later taken back into the plant at the inlet, making the task of desalination more difficult. For this application we do not necessarily care about the wider dispersal of high salinity water, but rather achieving an efficient and accurate calculation of the salinity at the inlet. If salt is interpreted as a passive tracer in the fluid flow, we can model its advection and diffusion using (6) and quantify its concentration at the inlet using (43). Through goal-oriented mesh adaptation, we can construct meshes which well approximate the salinity at the inlet, whilst retaining relatively few DoFs. An idealised (time-dependent) tidally varying desalination plant outfall scenario is considered in Subsection 5.1 .

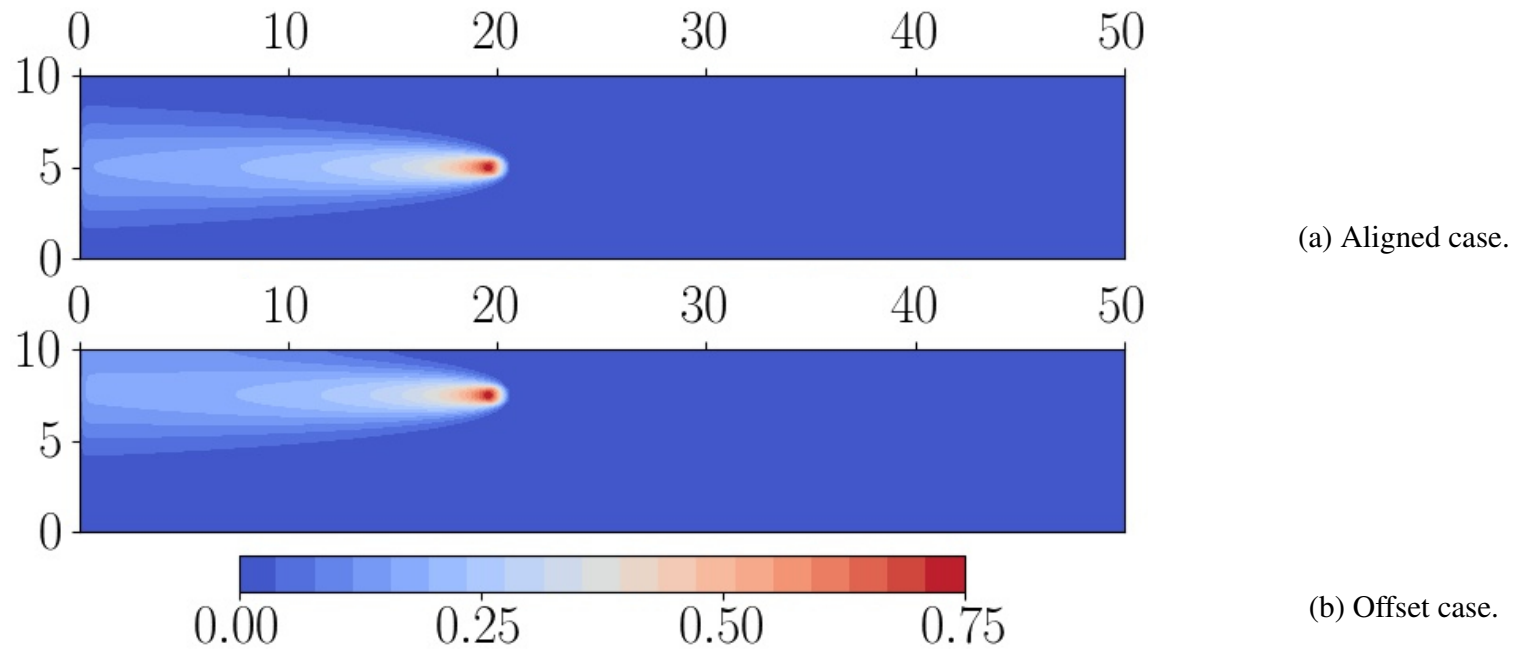

Figure 2: Discrete adjoint solutions for the 'Point Discharge with Diffusion' test case corresponding to QoIs of the form (43). Each field is presented in $\mathbb{P} 1$ space on a uniform mesh with 1,024,000 elements and 513,921 vertices. 
The derivative of the QoI integrand w.r.t. the tracer concentration provides a source term for the adjoint equation. In this case, the derivative is the indicator function $\mathbb{1}_{R_{i}}$, meaning the adjoint solution differs between the aligned and offset cases. Figure 2 presents discrete adjoint solutions associated with the SUPG stabilised forward problem.

In each case, we observe that, whilst the forward tracer concentration propagates downstream, the adjoint tracer concentration propagates upstream. The adjoint tracer concentration is near zero downstream of the receiver regions. This is consistent with the fact that the QoIs are independent of the downstream dynamics for advection-dominated problems.

\subsubsection{Application of Goal-Oriented Mesh Adaptation}

A finite element implementation has been presented and calibrated against an analytical solution. We now progress to perform goal-oriented error estimation and mesh adaptation within this setup, using the estimators and metrics described in Subsections 3.4 and 3.5 .
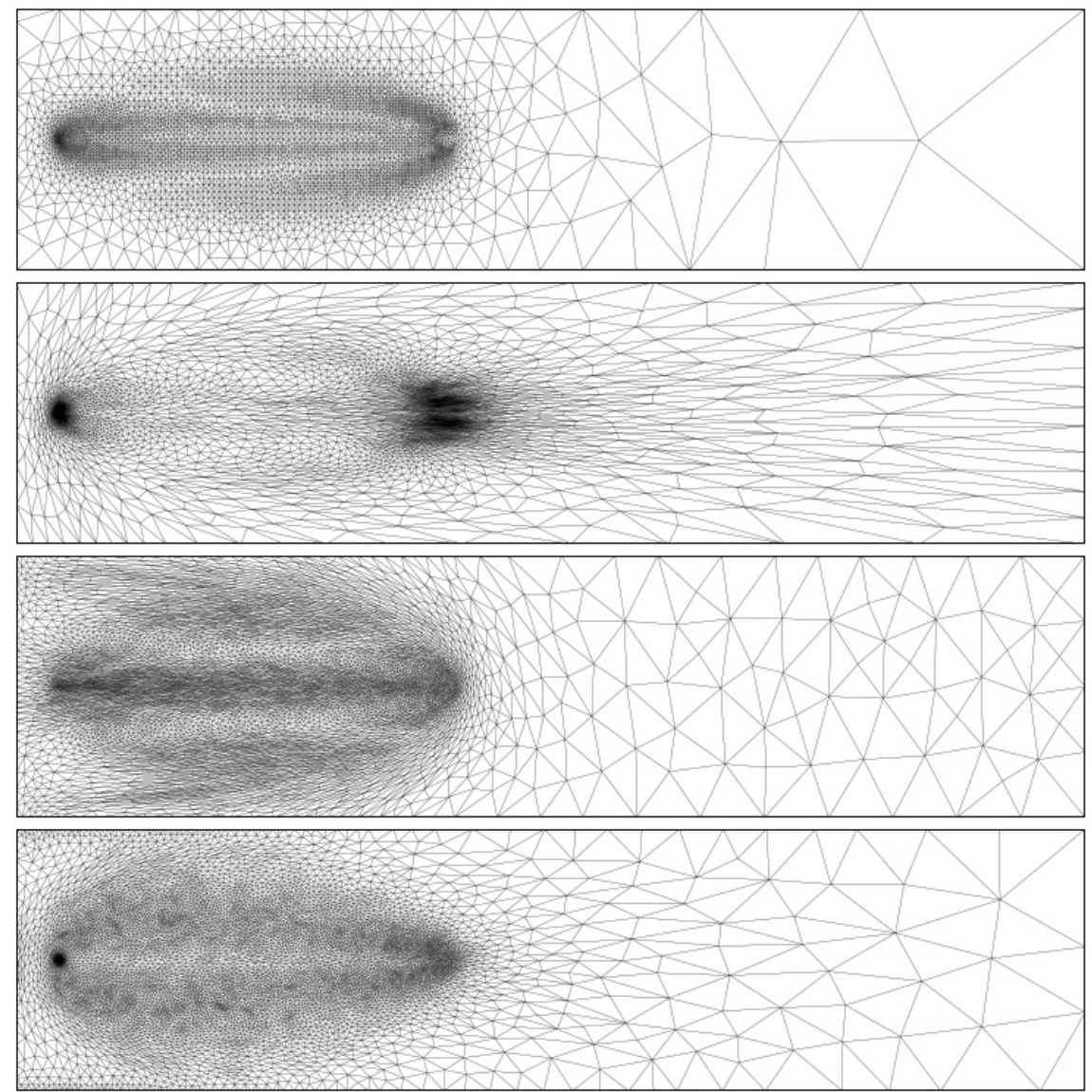

(a) Isotropic DWR $(11,273$ elements, 5,669 vertices, max. aspect ratio 2.7 )

(b) Anisotropic DWR $(13,269$ elements, 6,665 vertices, max. aspect ratio 20.3)

(c) Weighted Hessian $(11,419$ elements, 5,766 vertices, max. aspect ratio 24.1)

(d) Weighted gradient (17,658 elements, 8,918 vertices, max. aspect ratio 7.9)

Figure 3: Example meshes for the case of aligned source and receiver.

Figure 3 shows example meshes for the four goal-oriented metric generation methods, in the case of aligned source and receiver. The element count and maximum element aspect ratio of the mesh in each subfigure are stated in its caption. Each mesh has coarse mesh resolution downstream of the receiver region. This is in line with the fact that the QoI value is insensitive to the downstream dynamics. The majority of the mesh resolution lies in the region between source and receiver.

As may be expected, the isotropic DWR metric consists of triangles with relatively low aspect ratios (maximum 2.7), whilst the anisotropic metrics contain higher aspect ratios. Meshes due to the anisotropic DWR and WH approaches have notably high anisotropy. The maximum aspect ratios 20.3 and 24.1 observed in Subfigures $3 \mathrm{~b}$ and $3 \mathrm{c}$ are an order of magnitude higher than in the isotropic case. The former plot retains anisotropy downstream, whereas the latter has fairly isotropic elements downstream. The WH is isotropic downstream because it inherits anisotropy from the Hessian of the adjoint solution, which is uniformly zero in that region. The WG approach yields elements of moderate 
anisotropy across the entire domain, because its anisotropy is inherited from the Hessian of the potential, which is not uniformly zero. For the choices of normalisation parameters presented in Figure 3, observe that the anisotropic DWR and WG approaches tend to focus more mesh resolution around the source region than the others. The former also deploys significant mesh resolution surrounding the receiver region. The WG likely deploys high resolution at the source due to the additional term included in the metric in (31).
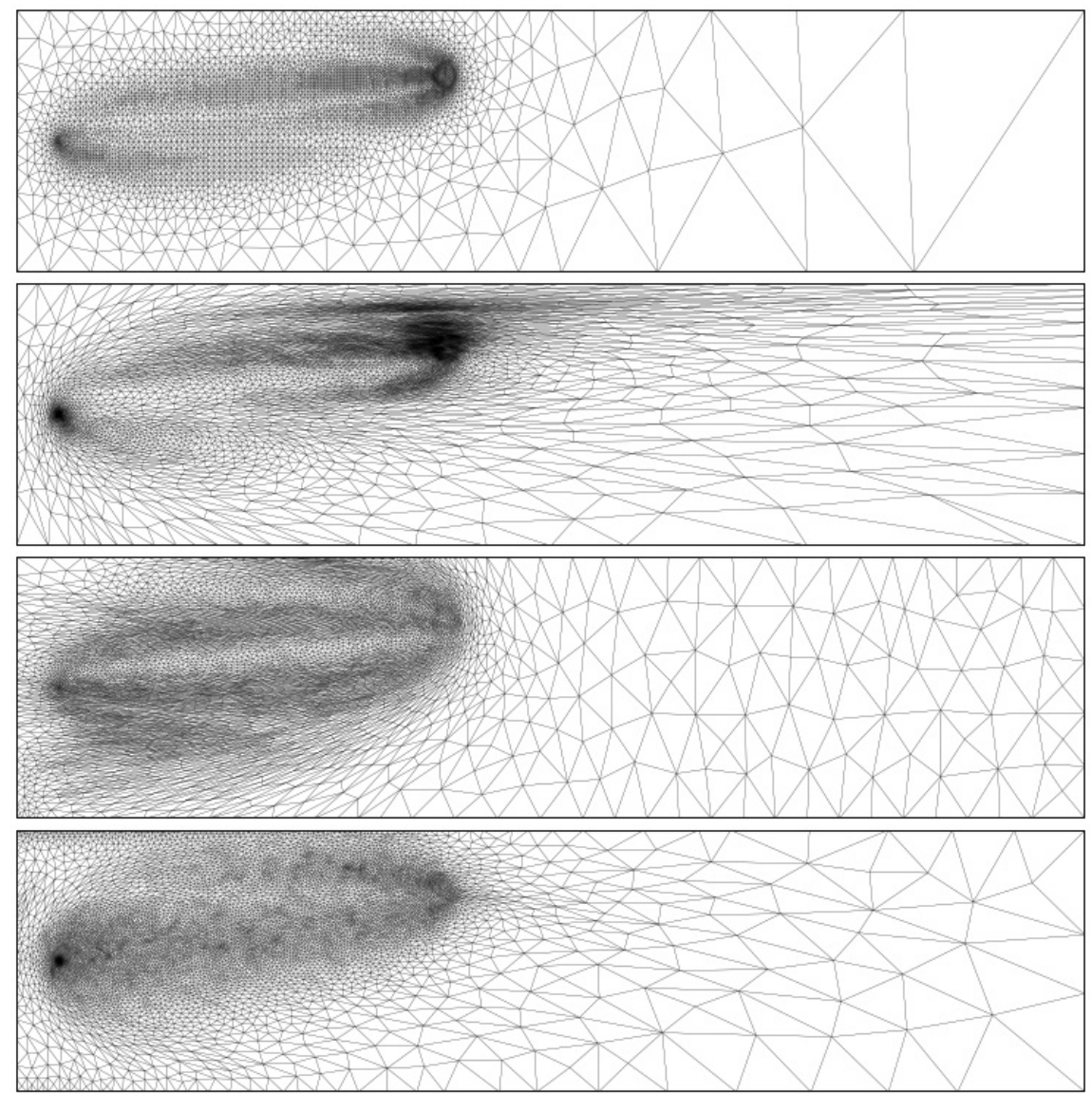

(a) Isotropic DWR $(11,246$ elements, 5,659 vertices, max. aspect ratio 1.6)

(b) Anisotropic DWR (13,067 elements, 6,566 vertices, max. aspect ratio 59.2)

(c) Weighted Hessian (10,877 elements, 5,507 vertices, max. aspect ratio 24.4)

(d) Weighted gradient $(17,803$ elements, 9,017 vertices, max. aspect ratio 7.0)

Figure 4: Example meshes for the case of offset source and receiver.

Figure 4 shows example meshes for the four goal-oriented metric generation methods, in the case of offset source and receiver. The same qualitative observations as made above can be applied again for the meshes in the offset case. An even larger maximum aspect ratio of 59.2 is reported for the anisotropic DWR metric. This is likely due to the skew introduced by the offset receiver.

There is one feature of the mesh due to the weighted gradient method which is clearer in the offset case than the aligned case. In generating the meshes presented in Subfigures $3 \mathrm{~d}$ and $4 \mathrm{~d}$, the 'volume' and 'surface' metrics 28 are intersected on the top and bottom boundary, where Neumann conditions are imposed. The surface metric includes information on how well these boundary conditions are satisfied. The mesh in Subfigure 4d clearly exhibits increased mesh refinement on the top boundary in the upstream region, compared with the bottom boundary. As well as including information on boundary condition error, the weighting by the adjoint solution means that fine mesh resolution is only deployed in regions where the QoI value is sensitive to perturbations in the forward solution. This is not the case on the bottom boundary, as may be deduced from Subfigure $2 \mathrm{~b}$.

Figure 5 shows relative error convergence curves for the QoI error in both aligned and offset cases, evaluated against the converged values established in the calibration experiment. Whilst we could consider convergence with increasing element count, we opt to measure mesh size using the number of vertices, because this equates to the DoF count in $\mathbb{P} 1$ space.

In each case, we observe that over 100,000 DoFs are required for repeated uniform refinement of the initial uniform mesh to yield less than $1 \%$ error. All four goal-oriented approaches also exhibit convergence to these values. However, in each adaptive case, the $1 \%$ error level is crossed using an order of magnitude fewer mesh DoFs. That is, with over 


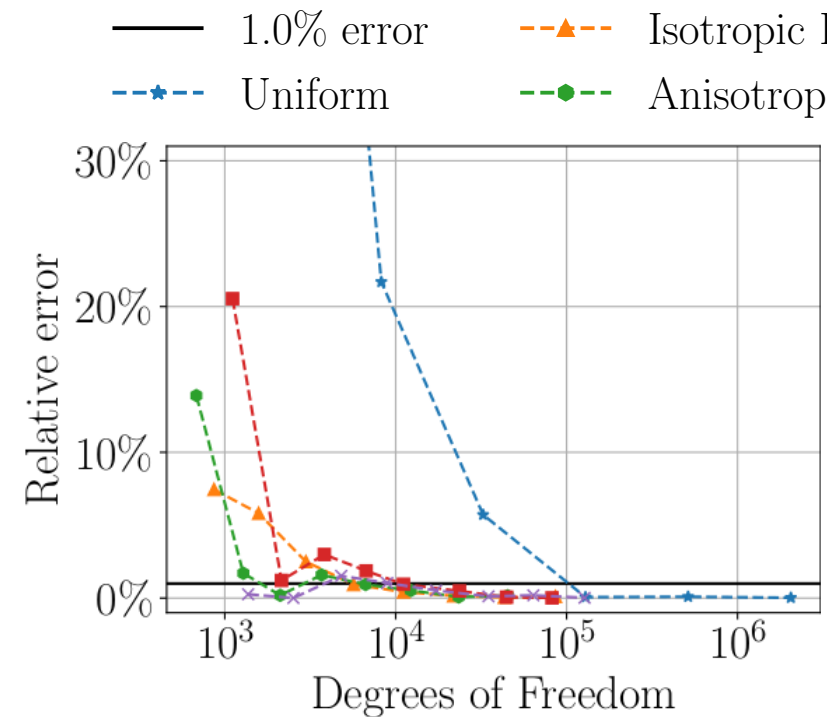

(a) Aligned source and receiver
----- Weighted Hessian

$--*--$ Weighted Gradient

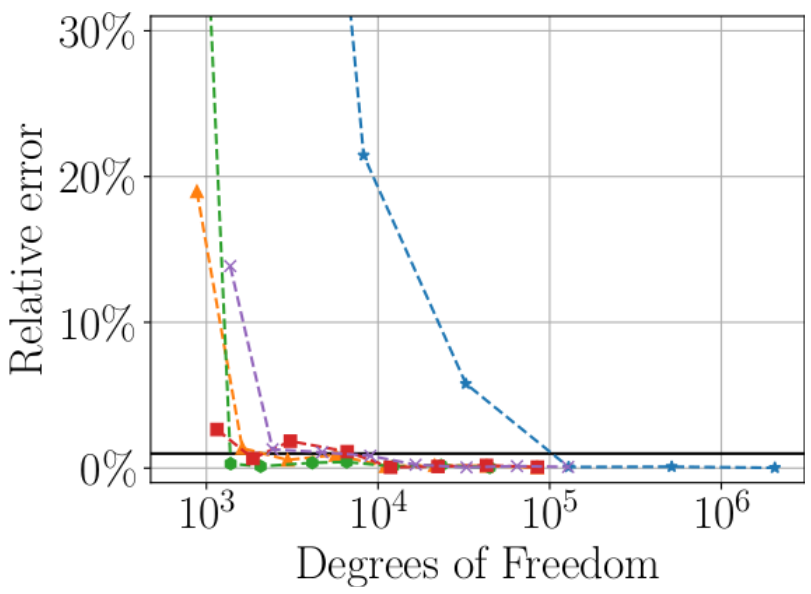

(b) Offset source and receiver

Figure 5: Convergence plots of relative QoI error against DoFs in the underlying $\mathbb{P} 1$ space.

10,000 DoFs, the adaptive strategies consistently yield QoI errors smaller than $1 \%$. It is interesting to observe that, whilst the meshes take rather different forms, all four approaches yield improved convergence properties with errors of comparable magnitude. The differences between convergence curves in Subfigure 5a are not significant enough to draw conclusions regarding optimality of one particular metric. In the offset case presented in Subfigure 5b, the adaptive methods again perform well, with all four yielding less than $1 \%$ error with 10,000 DoFs.

Remarkably, there are examples of meshes which allow for QoI error smaller than $1 \%$ with even fewer DoFs than in the 2,121 in the base mesh. See, for example, the WG metric in the aligned case and the anisotropic DWR metric in both aligned and offset cases. That this is possible without using enrichment methods to approximate the adjoint error demonstrates the effectiveness of the difference quotient indicator formulation. This formulation makes for a much more computationally efficient goal-oriented mesh adaptation strategy than solving the adjoint problem in a globally enriched space, as in [13]. Other improvements on that work are that the experiments presented in this section use a formulation of the WG metric which accounts for boundary terms, stabilisation errors are here accounted for in the WH metric and an anisotropic cell size measure was used.

\subsection{Point Discharge Test Case in Three Dimensions}

The previous subsection validated four metric-based goal-oriented mesh adaptation strategies for a 2D steady-state tracer transport problem. These metrics can also be readily applied in 3D. We demonstrate this by a simple extension of the 'Point Discharge with Diffusion' test case. Consider now the cuboid domain, $\Omega=[0,50] \times[0,10] \times[0,10] \mathrm{m}^{3}$, source region centred at $\mathbf{x}_{0}=(2,5,5)$ and offset receiver, $\mathbf{x}_{R}=(20,7.5,2.5)$. Fluid velocity remains solely in the positive $x$-direction and constant isotropic diffusion is again applied. The initial mesh is comprised of 240,000 uniform tetrahedra.

Figure 6 shows the tracer solution field computed on the mesh given by one uniform $h$-refinement of the base mesh. Observe that, whilst the profile is generally fairly smooth, the source term is not well captured. Further, unwanted element imprinting can be seen in the region surrounding the source.

Figure 7 illustrates a mesh generated using the WH approach, along with the associated tracer solution field. Where the source is not well captured on the uniform mesh, it appears to be much better resolved in on the adapted mesh, despite it having fewer elements overall. As in the 2D case, the mesh resolution due to the weighted Hessian metric is highest inbetween source and receiver, with low resolution deployed downstream. Some numerical diffusion is apparent downstream of the receiver in the contour plot, due to the very large elements there, but this is not of concern in an advection-dominated problem. 


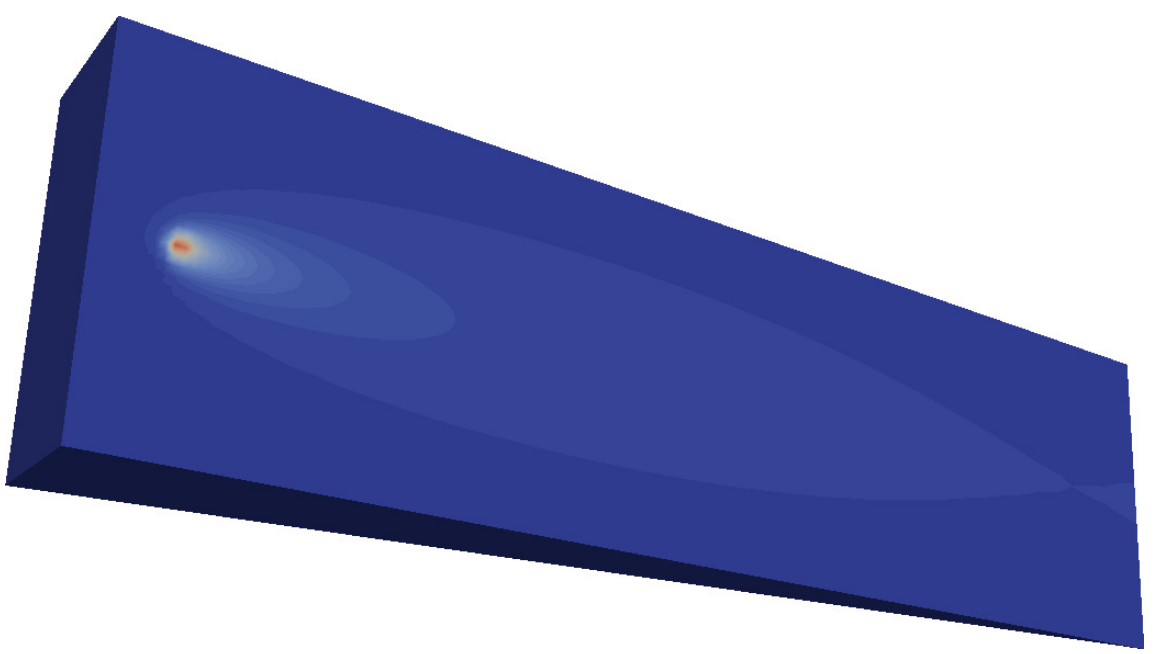

Figure 6: Tracer field computed on a uniform mesh with 1,920,000 tetrahedral elements and 337,881 vertices.
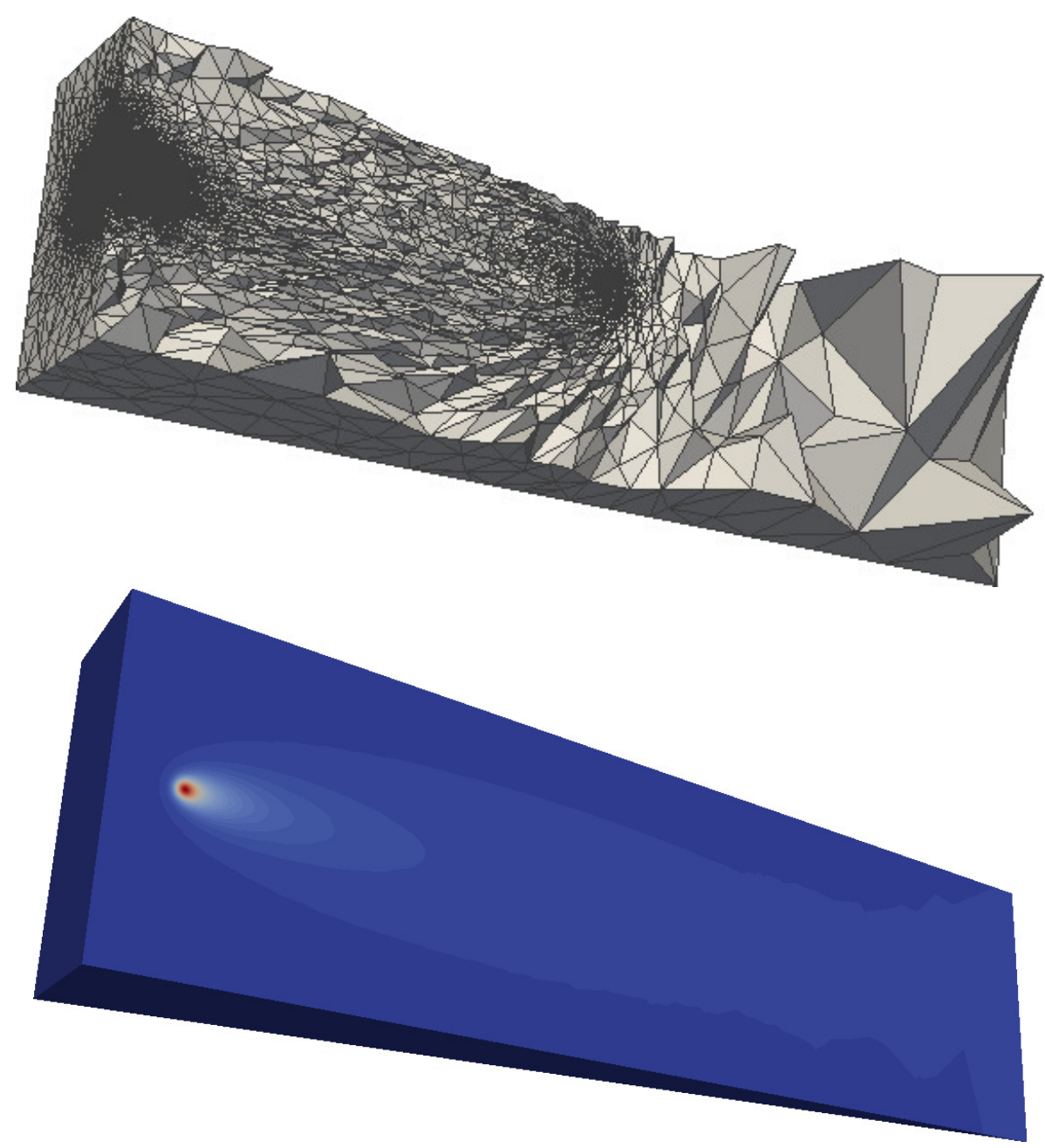

Figure 7: Example mesh generated using the weighted Hessian metric and the corresponding tracer field computed on this mesh (1,147,545 elements, 197,159 vertices, max. aspect ratio 13.6). 
The mesh presented in Figure 7 has maximum element volume 122.6 and minimum volume $1.68 \times 10^{-7}$, meaning that we have a truly multi-scale mesh. Multi-scale meshes are important in desalination plant outfall modelling, because the pipe diameters, bathymetric features and tidal forcings all exist on different orders of magnitude.

\section{Time-dependent Goal-Oriented Mesh Adaptation}

We have already seen that there exist many different ways to construct Riemannian metrics from goal-oriented error estimators for steady-state problems. Moving to the time-dependent case involves making more design choices. The first is how frequently the mesh should be adapted. Whilst frequent adaptation enables close tracking of dynamical features in the flow, it comes with increased interpolation error and additional computational overheads associated with the number of adaptation steps and the computation of the required metric tensors.

Extending to the time-dependent case means adding a time derivative term in (6):

$$
\left\{\begin{array}{rlrl}
\frac{\partial c}{\partial t}+\mathbf{u} \cdot \nabla c-\nabla \cdot(\underline{\boldsymbol{D}} \nabla c) & =S & & \text { in } \Omega \\
\underline{\boldsymbol{D}} \nabla c \cdot \widehat{\mathbf{n}} & =g_{N} & & \text { on } \partial \Omega_{N} \\
c & =g_{D} & & \text { on } \partial \Omega_{D}
\end{array} .\right.
$$

Where previously the fluid velocity $\mathbf{u}$ was prescribed as a constant, here it is time-dependent. In practice, it is usually given by the output of a hydrodynamics model. Under a depth-averaged approximation, for example, boundary forcings due to tides perturb the free surface elevation, $\eta$, which in turn drives $\mathbf{u}$. We interpret the tracer field $c$ as passive, meaning that it does not feed back into the hydrodynamics. As such, we perform goal-oriented error estimation for the tracer model only. For its application to Thetis' shallow water component, see [18].

We begin by decomposing the time period $(0, T]$ into a disjoint union of $k$ subintervals,

$$
(0, T]=\cup_{j=1}^{k} \mathcal{W}^{j}, \quad \mathcal{W}^{j}:=\left(t^{j-1}, t^{j}\right], \quad 0=t^{0}<t^{1}<\cdots<t^{k}=T .
$$

A mesh, $\mathcal{H}^{j}$, is associated with each subinterval, $\mathcal{W}^{j}$. As iterations of the adaptation loop progress, these meshes are updated, as indicated by a subscript index, $\mathcal{H}_{i}^{j}$. For simplicity, assume a constant timestep, $\Delta t>0$, and that each subinterval is of the same fixed length.

Given the temporal decomposition (45), one possible extension of Algorithm 1 to the time-dependent case is presented in Algorithm 2. For each iteration of the fixed point iteration loop, we again solve the forward and adjoint problems and use their solutions to construct metrics and thereby perform mesh adaptation. Note that there is an initial forward solve over the whole time period. In general, this requires interpolation of solution fields between meshes. For this, we use conservative projection [45], enabled in Firedrake by libsupermesh [46, 47]. Solution data are stored during the first forward solve, in order to provide initial conditions for the forward equations as solved on each subinterval in reverse.

Since we have a sequence of metrics and a target space-time complexity, this involves space-time normalisation, for which we use $\mathcal{L}_{p}$ normalisation as in formula (5). The approach is based on that proposed in [16, 17], which uses WG type metrics. Here we opt to use the WH metric.

Recall that goal-oriented error indicator (17) for the steady-state tracer transport problem is comprised of strong residual, boundary, flux and stabilisation terms. In the time-dependent case, it is more complicated. Applying Crank-Nicolson (for example) over timestep $\left(t^{(n)}, t^{(n+1)}\right]$ yields

$$
\begin{aligned}
\left.\rho^{(n)}\left(c_{h}, e^{*}\right)\right|_{K} & :=\frac{1}{\Delta t}\left\langle c_{h}^{(n+1)},\left.\left(e^{*}\right)\right|_{t=t^{(n+1)}}\right\rangle_{K}-\frac{1}{\Delta t}\left\langle c_{h}^{(n)},\left.\left(e^{*}\right)\right|_{t=t^{(n)}}\right\rangle_{K} \\
& -\left.\frac{1}{2} \rho\left(c_{h}^{(n+1)},\left.\left(e^{*}\right)\right|_{t=t^{(n+1)}}\right)\right|_{K}-\left.\frac{1}{2} \rho\left(c_{h}^{(n)},\left.\left(e^{*}\right)\right|_{t=t^{(n)}}\right)\right|_{K},
\end{aligned}
$$

That is, we have contributions from each time level, as well as a time derivative term. For the WH metric, we choose to interpret the adjoint error as the average of its values at times $t^{(n)}$ and $t^{(n+1)}$, for simplicity. Consequently, this metric averages the Hessians of the adjoint solutions from these timesteps and weights by the strong residual over the timestep $\left(t^{(n)}, t^{(n+1)}\right]$.

Note that for this time-dependent simulation, the continuous adjoint method is applied. This is because the application of the discrete adjoint method involves differentiating through mesh-to-mesh interpolations, which is not currently supported in Firedrake.

\subsection{Idealised Desalination Outfall Scenario}

The final numerical experiment in this paper considers desalination outfall in an idealised tidally varying channel. Consider the rectangular domain $\Omega=[-1500,1500] \times[-500,-500] \mathrm{m}^{2}$. For simplicity, the North and South 


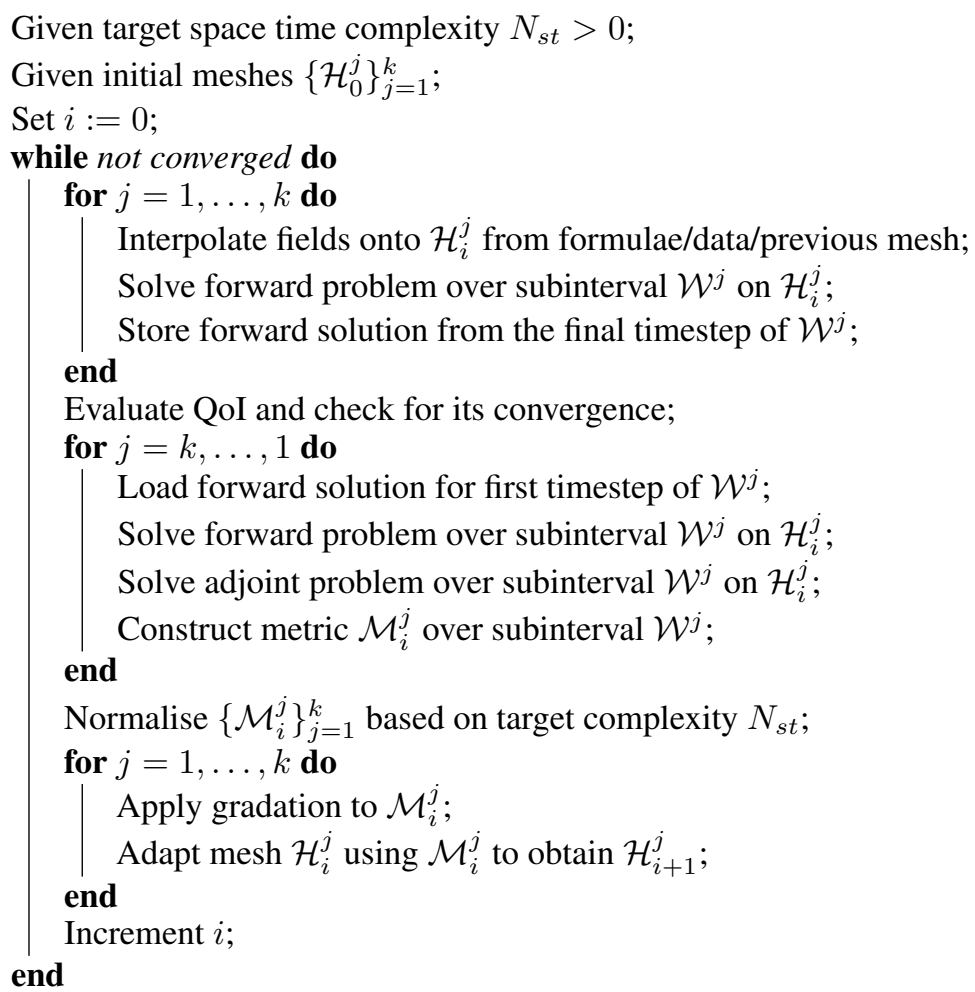

Algorithm 2: Mesh adaptation routine for time-dependent problems.

boundaries, $\partial \Omega_{N}$, are to be interpreted as impassible cliffs. Tidal forcings are imposed on the water surface elevation on the left and right boundaries, $\partial \Omega_{D}$. There, the salinity is set to a constant background value, $c_{b}=39 \mathrm{~g} \mathrm{l}^{-1}$.

Assuming constant bathymetry, viscosity and drag parameters and simple sinusoidal boundary forcings for the surface elevation, the hydrodynamics over the whole domain may be approximated using a sinusoidal forcing for the $x$ component of the velocity:

$$
\mathbf{u}(\mathbf{x}, t):=(U \sin (\omega t), 0), \quad \omega=\frac{2 \pi}{T_{\text {tide }}}, \quad U=1.15 \mathrm{~m} \mathrm{~s}^{-1}
$$

Doing so means that we only need to solve a linear PDE (the tracer transport system) at each timestep, implying a low computational cost for the forward solve. For the purposes of this numerical experiment, $T_{\text {tide }}$ is set to $5 \%$ of the $\mathrm{M} 2$ tidal constituent, meaning it is artificially sped up twenty-fold. We choose a timestep of $\Delta t=2.232$, so that there are 2,000 timesteps in total. The time interval is divided into 100 uniform subintervals.

The desalination plant has buried pipes with open ends located within the domain. The outlet pipe has diameter $r_{\text {out }}=25 \mathrm{~m}$ and is positioned at $\mathbf{x}_{\text {out }}=(0,100)$. The inlet pipe also has diameter $r_{\text {in }}=25 \mathrm{~m}$, but is positioned at $\mathbf{x}_{\mathrm{in}}=(400,-100)$. In the terminology and notation of the previous experiments, a source is released from $R_{\text {out }}:=B_{r_{\text {out }}}\left(\mathbf{x}_{\text {out }}\right)$ and received in $R_{\text {in }}:=B_{r_{\text {in }}}\left(\mathbf{x}_{\text {in }}\right)$. The injection of saline water into the flow is modelled by the circular indicator function $\mathbb{1}_{R_{\text {out }}}$, scaled by discharge rate of 2 .

Tracer dispersion is modelled over two tidal periods. The QoI is given as the increase in salinity at the inlet pipe, against the background value:

$$
J(c):=\int_{0}^{2 T_{\text {tide }}} \int_{\Omega} \mathbb{1}_{R_{\text {in }}}\left(c-c_{b}\right) \mathrm{d} x \mathrm{~d} t=\int_{0}^{2 T_{\text {tide }}} \int_{R_{\text {in }}}\left(c-c_{b}\right) \mathrm{d} x \mathrm{~d} t .
$$

The linearity of the QoI and forward equation mean that the adjoint problem is independent of the forward solution. This implies there is no need to checkpoint state.

Figure 8 displays snapshots of the adaptive mesh and salinity due to the WH approach at five time levels, including the initial and final times.

At $t=0$, the tracer concentration is initialised to the background level. The mesh at that time is adapted to the WH computed over the following 20 timesteps, which is why there is increased mesh resolution around the outlet pipe. 


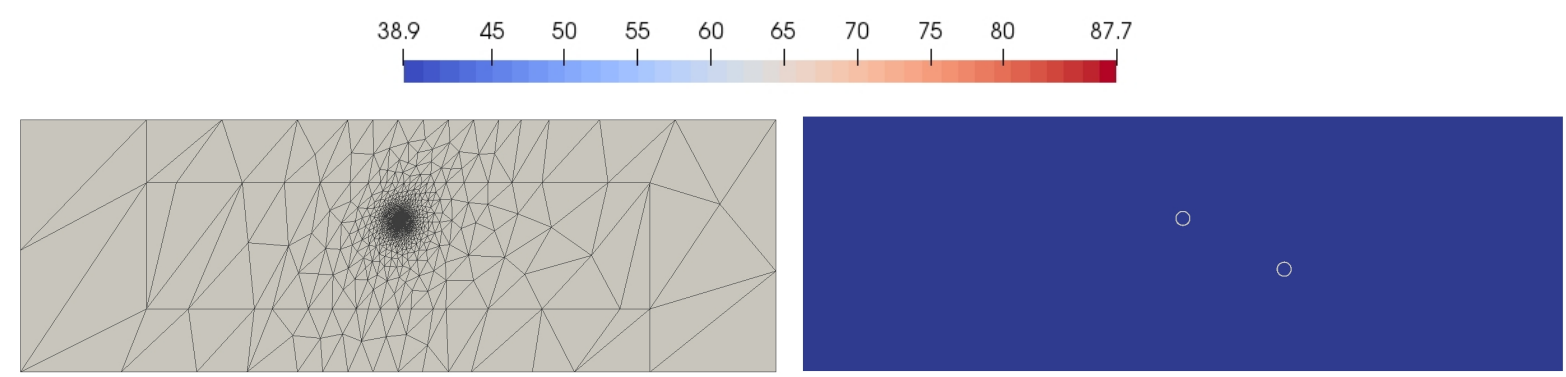

(a) $t=0 \mathrm{~s}(2,304$ elements, 1,170 vertices, max. aspect ratio 3.1$)$
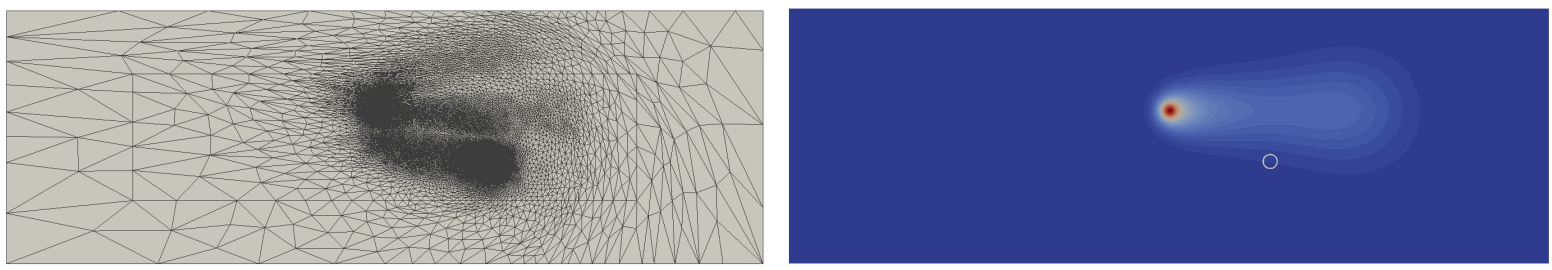

(b) $t=500 \mathrm{~s}(42,696$ elements, 21,380 vertices, max. aspect ratio 9.0)
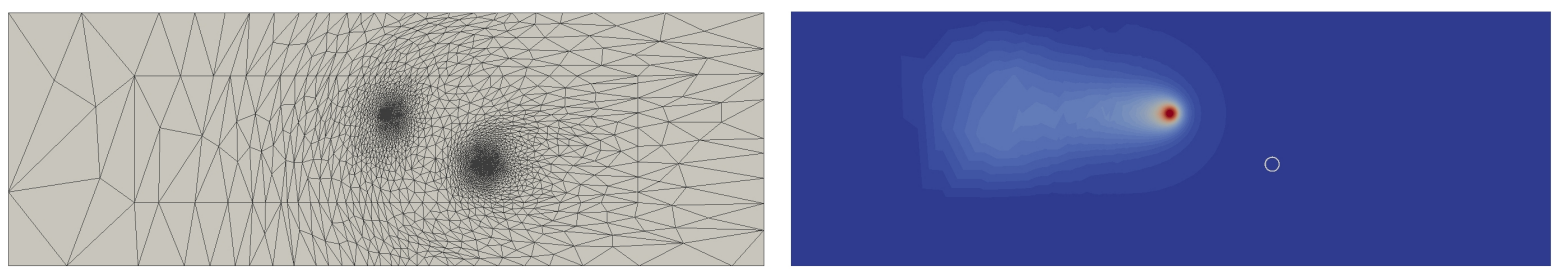

(c) $t=1,000 \mathrm{~s}(5,306$ elements, 2,687 vertices, max. aspect ratio 6.3)
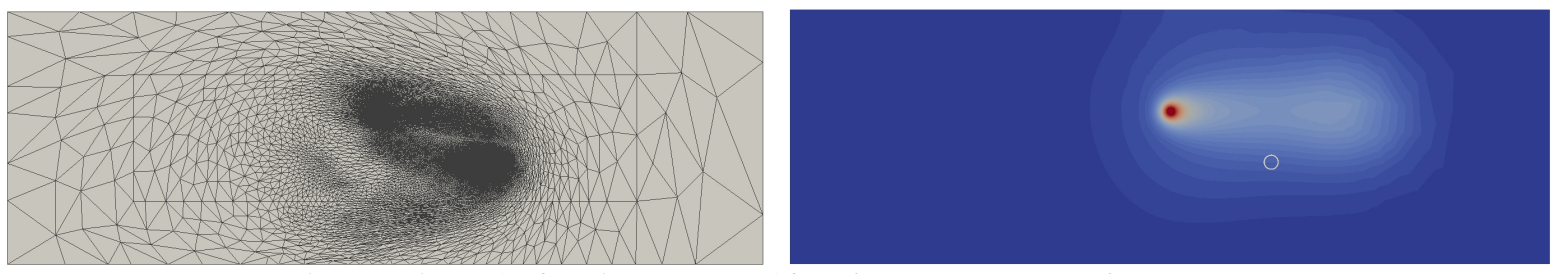

(d) $t=1,500 \mathrm{~s}(41,627$ elements, 20,846 vertices, max. aspect ratio 17.7)
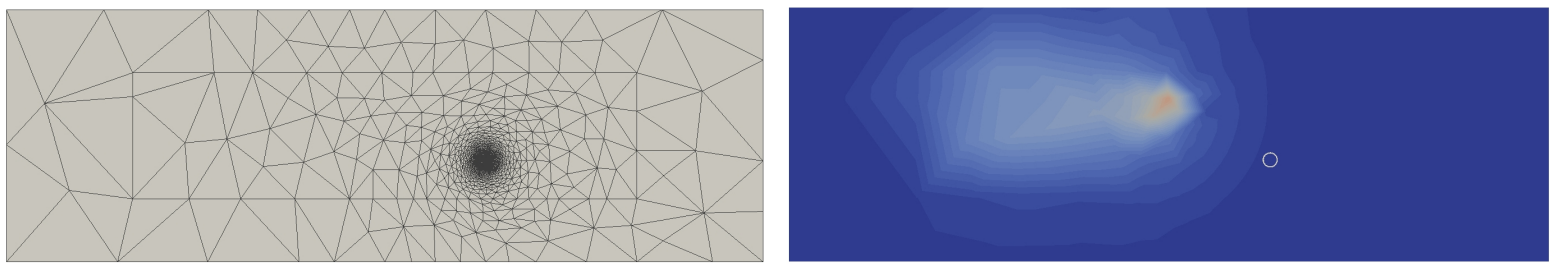

(e) $t=2,000 \mathrm{~s}(2,409$ elements, 1,222 vertices, max. aspect ratio 3.2)

Figure 8: Example meshes for the the application of the weighted Hessian metric with normalisation order $p=10$ to the idealised desalination problem. The two circles in Subfigure 8a indicate the locations of the inlet and outlet pipes, with radii to scale. The single circle in each of the other subfigures indicates the inlet pipe.

Similarly, the mesh at the final time $t=2,000$ is only well resolved around the inlet. This is because saline water released from the outlet will not reach it before the end of the simulation, meaning the adjoint solution is negligible away from the inlet. Indeed, the tracer concentration away from the inlet has suffered high levels of artificial numerical diffusion, since it is not important with regards to the goal and so is represented using coarse mesh resolution. Numerical diffusion can also be seen where the tracer concentration meets coarse mesh resolution at time level $t=1,000$.

At $t=500$ and $t=1,500$, the isolines are relatively smooth - in line with the high mesh resolution deployed between outlet and inlet - although some numerical diffusion is apparent downstream in Subfigure 8d. The meshes at these time levels have approximately an order of magnitude more DoFs than is apparent in the other snapshots. This observation 
reveals how the goal-oriented mesh adaptation can be used to construct discretisations which are multi-scale in time, as well as in space. The mechanism which allows for such features to develop is the space-time normalisation strategy, which distributes goal-oriented metric complexity across the subintervals.

As well as having more DoFs, the meshes at times $t=500$ and $t=1,500$ (particularly the latter) have higher anisotropy than the others presented. This is due to constructive interference between contributions from the strong residual and the Hessian of the adjoint solution.

\section{Discussion}

\subsection{Conclusion}

This work presents the successful implementation of four approaches to goal-oriented mesh adaptation in the finite element package Firedrake. Earlier versions of three of the approaches were previously compared in Firedrake in [13] and the other was used in [18]. Many of the simplifications of the former implementation have been overcome in this work and a stabilisation strategy which accounts for mesh anisotropy has been deployed.

The numerical experiments presented in Section 4 use an established advection-diffusion test case with a known analytical solution to validate the goal-oriented strategies, demonstrating convergence of two quantities of interest under increasing DoF count. Convergence is achieved using at least an order of magnitude fewer elements than under uniform refinement, for each adaptation strategy. Clear conclusions cannot be drawn from this simple test case regarding the optimality of any one of the methods. However, the comparison is useful for identifying the ways in which each strategy deploys mesh resolution in order to better capture the quantity of interest. Subsection 4.3 demonstrates the extension of the goal-oriented mesh adaptation framework to the three dimensional case, wherein a spatially multi-scale mesh is presented.

The experiments in this paper use continuous finite element methods, meaning that flux terms only arise in error indicators due to the integration by parts. However, the 'isotropic DWR metric' and 'anisotropic DWR metric' readily extend to discontinuous finite element methods, as investigated in [18]. Whilst the 'weighted Hessian' metric introduced in [14] may be modified to account for stabilisation methods (as shown in Subsection 3.5), the metric will require further modification in order to account for errors due to discontinuous discretisations. Since the 'weighted gradient' is based on an a priori error result, it is discretisation agnostic.

The experiments in Section 4 act as a foundation, upon which more complex, realistic problems may be considered. A key achievement of this particular work is the extension of the goal-oriented mesh adaptation framework to the time-dependent case. In Section 5, this extension is applied to a tidally-varying desalination plant outfall scenario. For an idealised setup, goal-oriented mesh adaptation is successfully applied. Snapshots of meshes and salinity fields are presented, revealing that goal-oriented mesh adaptation can yield discretisations which are multi-scale in time as well as space.

\subsection{Outlook}

In the idealised desalination outfall setup, tidal hydrodynamics were approximated using a sinusoidal velocity field. For realistic problems, complex bathymetry fields, drag coefficients and turbulent flow features mean that a prescribed velocity is insufficient; instead, a hydrodynamics model should be included in the forward problem. Hydrodynamics models are typically nonlinear and come with higher computational costs than solving the tracer transport equation alone. At the very least, the fluid velocity should be computed during the forward solve, stored and then loaded again during the adjoint solve. This is quite feasible for the relatively small problem sizes considered in this paper. However, for large-scale problems with highly resolved features, solution fields at each timestep can require significant amounts of memory, meaning storing the entire trajectory becomes infeasible. In such a situation, it becomes necessary to use checkpointing routines (such as [48]), which make a trade-off between recomputing timesteps and storing the associated data.

In this paper, salinity is treated as a passive tracer in a barotropic ocean. In the baroclinic case, density is not simply a function of pressure and depends on salinity, as well as temperature. That is, salinity is an active tracer. Future work will apply goal-oriented mesh adaptation to such flows, in which case the computational cost associated with forward and adjoint solves is significant and checkpointing routines are necessary. 


\section{Acknowledgements}

Many thanks to members of Imperial College London's Applied Modelling and Computation Group (AMCG) and to the developers of the Firedrake and Thetis projects for their useful recommendations regarding this work. Further thanks to staff and students of the Mathematics of Planet Earth Centre for Doctoral Training (MPE CDT) for their ongoing support and advice. Funding: This work was supported by the Engineering and Physical Sciences Research Council (EPSRC) [grant numbers EP/L016613/1, EP/R029423/1].

\section{References}

[1] R. Becker, R. Rannacher, A feed-back approach to error control in finite element methods: basic analysis and examples, IWR, 1996.

[2] R. Becker, R. Rannacher, An optimal control approach to a posteriori error estimation in finite element methods, Acta numerica 10 (2001) 1-102.

[3] L. Formaggia, S. Micheletti, S. Perotto, Anisotropic mesh adaptation in computational fluid dynamics: application to the advection-diffusion-reaction and the Stokes problems, Applied Numerical Mathematics 51 (4) (2004) 511-533.

[4] P. Houston, E. H. Georgoulis, E. Hall, Adaptivity and a posteriori error estimation for DG methods on anisotropic meshes (2006).

[5] J. Carpio, J. L. Prieto, R. Bermejo, Anisotropic "goal-oriented" mesh adaptivity for elliptic problems, SIAM Journal on Scientific Computing 35 (2) (2013) A861-A885.

[6] V. Dolejší, F. Roskovec, Goal-oriented error estimates including algebraic errors in discontinuous Galerkin discretizations of linear boundary value problems, Applications of Mathematics 62 (6) (2017) 579-605.

[7] V. Dolejší, G. May, A. Rangarajan, F. Roskovec, A goal-oriented high-order anisotropic mesh adaptation using discontinuous Galerkin method for linear convection-diffusion-reaction problems, SIAM Journal on Scientific Computing 41 (3) (2019) A1899-A1922.

[8] P. George, F. Hecht, M. Vallet, Creation of internal points in Voronoi's type method. Control and adaptation, Adv. Eng. Software 13 (5-6) (1991) 303-312.

[9] C. Pain, A. Umpleby, C. De Oliveira, A. Goddard, Tetrahedral mesh optimisation and adaptivity for steady-state and transient finite element calculations, Computer Methods in Applied Mechanics and Engineering 190 (29-30) (2001) 3771-3796.

[10] F. Alauzet, G. Olivier, An $L^{p}-L^{\infty}$ space-time anisotropic mesh adaptation strategy for time dependent problems, in: Proceedings of ECCOMAS CFD, 2010.

[11] A. Loseille, F. Alauzet, Continuous mesh framework part I: well-posed continuous interpolation error, SIAM Journal on Numerical Analysis 49 (1) (2011) 38-60.

[12] M. D. Piggott, P. E. Farrell, C. R. Wilson, G. J. Gorman, C. C. Pain, Anisotropic mesh adaptivity for multi-scale ocean modelling, Philosophical Transactions of the Royal Society A: Mathematical, Physical and Engineering Sciences 367 (1907) (2009) 4591-4611. doi:10.1098/rsta.2009.0155

[13] J. G. Wallwork, N. Barral, D. A. Ham, M. D. Piggott, Anisotropic goal-oriented mesh adaptation in Firedrake in: 28th Intl Meshing Roundtable, Zenodo, 2020, pp. 83-100. doi:10.5281/zenodo.3653101 URL https://doi.org/10.5281/zenodo.3653101

[14] P. Power, C. C. Pain, M. Piggott, F. Fang, G. J. Gorman, A. Umpleby, A. J. Goddard, I. Navon, Adjoint a posteriori error measures for anisotropic mesh optimisation, Computers \& Mathematics with Applications 52 (8-9) (2006) $1213-1242$.

[15] A. Loseille, A. Dervieux, F. Alauzet, Fully anisotropic goal-oriented mesh adaptation for 3D steady euler equations, Journal of computational physics 229 (8) (2010) 2866-2897.

[16] F. Alauzet, A. Belme, A. Dervieux, Anisotropic goal-oriented mesh adaptation for time dependent problems, in: 20th Intl Meshing Roundtable, Springer, 2011, pp. 99-121.

[17] A. Belme, A. Dervieux, F. Alauzet, Time accurate anisotropic goal-oriented mesh adaptation for unsteady flows, Journal of Computational Physics 231 (19) (2012) 6323-6348. 
[18] J. G. Wallwork, N. Barral, S. C. Kramer, D. A. Ham, M. D. Piggott, Goal-oriented error estimation and mesh adaptation for shallow water modelling, Springer Nature Applied Sciences 2 (2020) 1053-1063. doi:10.1007/ s42452-020-2745-9. URL https://rdcu.be/b35wZ

[19] F. Rathgeber, D. A. Ham, L. Mitchell, M. Lange, F. Luporini, A. T. McRae, G.-T. Bercea, G. R. Markall, P. H. Kelly, Firedrake: automating the finite element method by composing abstractions, ACM Transactions on Mathematical Software (TOMS) 43 (3) (2016) 24. doi :10.1137/15M1021167. URL http://arxiv.org/abs/1411.2940

[20] T. Kärnä, S. C. Kramer, L. Mitchell, D. A. Ham, M. D. Piggott, A. M. Baptista, Thetis coastal ocean model: discontinuous Galerkin discretization for the three-dimensional hydrostatic equations, Geoscientific Model Development 11 (11) (2018) 4359-4382. doi:10.5194/gmd-11-4359-2018

[21] Software used in 'Goal-Oriented Error Estimation and Mesh Adaptation for Tracer Transport Modelling' (Nov. 2020). doi: $10.5281 /$ zenodo.4293614.

URL https://doi.org/10.5281/zenodo.4293614

[22] T. Kärnä, S. C. Kramer, L. Mitchell, A. Angeloudis, J. G. Wallwork, M. C. A. Clare, D. A. Ham, N. Barral, A. T. T. McRae, S. Warder, thetisproject/thetis: Thetis coastal ocean model (Nov. 2020). doi:10.5281/zenodo 4288261 .

URL https://doi.org/10.5281/zenodo.4288261

[23] J. G. Wallwork, M. C. A. Clare, Simulation Code for 'Goal-Oriented Error Estimation and Mesh Adaptation for Tracer Transport Modelling' (Jan. 2021). doi:10.5281/zenodo.4468992

URL https://doi.org/10.5281/zenodo.4468992

[24] A. Riadh, G. Cedric, M. Jean, TELEMAC modeling system: 2D hydrodynamics TELEMAC-2D software release 7.0 user manual, Paris: R\&D, Electricite de France (2014) 134.

[25] F. Alauzet, Size gradation control of anisotropic meshes, Finite Elements in Analysis and Design 46 (1-2) (2010) 181-202.

[26] F. Alauzet, A. Loseille, A decade of progress on anisotropic mesh adaptation for computational fluid dynamics, Computer-Aided Design 72 (2016) 13-39.

[27] N. Barral, Time-accurate anisotropic mesh adaptation for three-dimensional moving mesh problems, Ph.D. thesis, Université Pierre et Marie Curie (2015).

[28] N. Barral, M. G. Knepley, M. Lange, M. D. Piggott, G. J. Gorman, Anisotropic mesh adaptation in Firedrake with PETSc DMPlex, arXiv preprint arXiv:1610.09874 (2016).

[29] D. Ibanez, N. Barral, J. Krakos, A. Loseille, T. Michal, M. Park, First benchmark of the unstructured grid adaptation working group, Procedia engineering 203 (2017) 154-166.

[30] M. A. Park, N. Barral, D. Ibanez, D. S. Kamenetskiy, J. A. Krakos, T. R. Michal, A. Loseille, Unstructured grid adaptation and solver technology for turbulent flows, in: 2018 AIAA Aerospace Sciences Meeting, 2018, p. 1103.

[31] M. A. Park, A. Balan, W. K. Anderson, M. C. Galbraith, P. Caplan, H. A. Carson, T. R. Michal, J. A. Krakos, D. S. Kamenetskiy, A. Loseille, et al., Verification of unstructured grid adaptation components, in: AIAA Scitech 2019 Forum, 2019, p. 1723.

[32] L. D. Dalcin, R. R. Paz, P. A. Kler, A. Cosimo, Parallel distributed computing using Python, Advances in Water Resources 34 (9) (2011) 1124-1139, new Computational Methods and Software Tools. doi:http: //dx.doi.org/10.1016/j.advwatres.2011.04.013

[33] S. Balay, S. Abhyankar, M. F. Adams, J. Brown, P. Brune, K. Buschelman, L. Dalcin, A. Dener, V. Eijkhout, W. D. Gropp, D. Kaushik, M. G. Knepley, D. A. May, L. C. McInnes, R. T. Mills, T. Munson, K. Rupp, P. Sanan, B. F. Smith, S. Zampini, H. Zhang, H. Zhang, PETSc users manual, Tech. Rep. ANL-95/11 - Revision 3.11, Argonne National Laboratory (2019). URL http://www.mcs.anl.gov/petsc

[34] S. Balay, W. D. Gropp, L. C. McInnes, B. F. Smith, Efficient management of parallelism in object oriented numerical software libraries, in: E. Arge, A. M. Bruaset, H. P. Langtangen (Eds.), Modern Software Tools in Scientific Computing, Birkhäuser Press, 1997, pp. 163-202.

[35] A. N. Brooks, T. J. Hughes, Streamline upwind/Petrov-Galerkin formulations for convection dominated flows with particular emphasis on the incompressible Navier-Stokes equations, Computer methods in applied mechanics and engineering 32 (1-3) (1982) 199-259. 
[36] S. Micheletti, S. Perotto, M. Picasso, Stabilized finite elements on anisotropic meshes: a priori error estimates for the advection-diffusion and the Stokes problems, SIAM Journal on Numerical Analysis 41 (3) (2003) 1131-1162.

[37] P. R. Amestoy, I. S. Duff, J.-Y. L'Excellent, J. Koster, A fully asynchronous multifrontal solver using distributed dynamic scheduling, SIAM Journal on Matrix Analysis and Applications 23 (1) (2001) 15-41.

[38] P. R. Amestoy, A. Guermouche, J.-Y. L'Excellent, S. Pralet, Hybrid scheduling for the parallel solution of linear systems, Parallel Computing 32 (2) (2006) 136-156.

[39] P. E. Farrell, D. A. Ham, S. W. Funke, M. E. Rognes, Automated derivation of the adjoint of high-level transient finite element programs, SIAM Journal on Scientific Computing 35 (4) (2013) C369-C393.

[40] M. D. Gunzburger, Perspectives in flow control and optimization, SIAM, 2002.

[41] P. Clément, Approximation by finite element functions using local regularization, Revue française d'automatique, informatique, recherche opérationnelle. Analyse numérique 9 (R2) (1975) 77-84.

[42] M. Braack, An adaptive finite element method for reactive-flow problems, Ph.D. thesis, IWR (1998).

[43] P. Ciarlet, The finite element method for elliptic problems (1978).

[44] C. Zhu, R. H. Byrd, P. Lu, J. Nocedal, Algorithm 778: L-BFGS-B: Fortran subroutines for large-scale boundconstrained optimization, ACM Transactions on Mathematical Software (TOMS) 23 (4) (1997) 550-560.

[45] P. Farrell, M. Piggott, C. Pain, G. Gorman, C. Wilson, Conservative interpolation between unstructured meshes via supermesh construction, Computer Methods in Applied Mechanics and Engineering 198 (33) (2009) 2632 2642. doi:https://doi.org/10.1016/j.cma.2009.03.004.

[46] P. E. Farrell, J. R. Maddison, Conservative interpolation between volume meshes by local Galerkin projection, Computer Methods in Applied Mechanics and Engineering 200 (1-4) (2011) 89-100. doi:10.1016/j.cma. 2010.07.015

[47] J. R. Maddison, P. E. Farrell, I. P. Panourglas, Parallel supermeshing for multimesh modelling, Tech. Rep. eCSE0308, ARCHER (May 2016). URL https://www.archer.co.uk/community/eCSE/eCSE03-08/eCSE03-08-TechnicalReport.pdf

[48] A. Griewank, A. Walther, Algorithm 799: revolve: an implementation of checkpointing for the reverse or adjoint mode of computational differentiation, ACM Transactions on Mathematical Software (TOMS) 26 (1) (2000) $19-45$. 